\title{
Weak Deflection Angle and Bounding Greybody of Electric and Magnetic Charged Black Holes in Nonlinear Electrodynamics
}

\author{
Wajiha Javed, ${ }^{1, *}$ Mehak Atique, ${ }^{1, \dagger}$ and Ali Övgün ${ }^{2, \ddagger}$ \\ ${ }^{1}$ Department of Mathematics, Division of Science and Technology, University of Education, Lahore-54590, Pakistan \\ ${ }^{2}$ Physics Department, Eastern Mediterranean University, \\ Famagusta, 99628 North Cyprus via Mersin 10, Turkey.
}

(Dated: February 17, 2022)

\begin{abstract}
In this paper, we study the weak deflection angle using Gauss-Bonnet theorem and bounding greybody factor for electric and magnetic black holes in the background of nonlinear electrodynamics. Using Gibbons and Werner's approach, first we acquire the Gaussian optical curvature to use in Gauss-Bonnet theorem and calculate the bending angle for spherically symmetric electric and magnetic black holes in both non-plasma and plasma mediums in the weak field limits. Later, we calculate the rigorous bounds of the greybody factor for the given black holes. Furthermore, we look into the graphical behaviour of bending angles and greybody bounds at some specific values of multiple parameters as well as black holes charges. It is to be mention here that all the results for the electric and magnetic charged black holes solutions reduce into the Schwarzschild black hole solution in the absence of the black holes charges.
\end{abstract}

PACS numbers: 95.30.Sf, 98.62.Sb, 97.60.Lf

Keywords: Gravitational Lensing; Black Holes; Gauss-Bonnet Theorem; Plasma Medium; Greybody Factor; Nonlinear electrodynamics

\section{INTRODUCTION}

Black holes (BHs) are one of the most fascinating objects in the universe. The presence of BHs has remained the subject of interest since Einstein found their existence in the universe using his theory of general relativity (GR). Black holes give a significant tool to examine and test the essential laws of the universe. A BH is an area in the universe that has such an excessive gravity that nothing can get away from its pull, even the light can not escape. Einstein declared the presence of gravitational waves and gravitational lensing as phenomena of GR in 1916 [1]. The gravitational waves from BHs and neutron stars merger had been recognized via LIGO, which proved the theoretical expectancies that are nicely geared up with experimental studies $[2,3]$. In addition, the first image of the $\mathrm{BH}$ was seen by Event Horizon Telescope collaboration [4, 5]. After the identification of the gravitational waves, a large wide variety of gravity theories faces many drawbacks, but the identification of the gravitational waves also grabbed the attention in the field of gravitational lensing (GL) [6]. The concept of GL was first presented by Soldner in 1801 within the history of Newton's theory of gravitation [7]. To grasp the knowledge of galaxies and universe, GL is extremely useful technique. At that point, facts taken in the duration of a solar eclipse for a rich star field in 1919, explorations via Eddington anticipations just about that of the GR estimations [8]. During the instant of solar eclipse, the astrophysicist observed bending within the starlight that have been as a result of the Sun's gravitational field. After this, in comparison of results with the Einstein predictions, they observed that they have got similar findings. These consequences performed the crucial role within the experiential evidence of Einstein's prediction. Gravitational lensing is the bending of light within the extant of gigantic objects which is expected by GR. In literature GL has been divided into three types (i) strong GL (ii) weak GL (iii) micro GL [9]-[14].

Weak lensing is an effective way for the measurement of the astronomical object which might be big (including $\mathrm{BH}$ ) and have huge radii without demanding about their structures. The expansion of the universe can also be studied by using GL. The study of GL has gained much attention from researchers. Gravitational lensing has proved very useful to investigate many astrophysical objects like naked singularities, BHs and the wormholes [9]-[20]. In past years, many investigations have connected GL with the Gauss-Bonnet theorem (GBT) after Gibbons and Werner confirmation about the feasible manner to calculate the bending angle from BHs that show asymptotic behaviour by using GBT [21]:

$$
\breve{\Theta}=-\iint_{D_{\infty}} \mathcal{K} d S
$$

\footnotetext{
*Electronic address: wajiha.javed@ue.edu.pk

${ }^{\dagger}$ Electronic address: mehakatique1997@gmail.com

‡Electronic address: ali.ovgun@emu.edu.tr
} 
Here, $\mathcal{K}$ is representing the optical Gaussian curvature and $d S$ represents optical surface. Werner extended this technique for stationary BHs using Kerr-Randers optical geometry [22]. Recently, Gallo and Crisnejo [23] examined the bending light in the plasma medium. Since then the interest within the work of weak lensing is continuously increasing each day through the method of Gibbons and Werner via use of the GBT for BHs [24]-[64].

In 1974, Hawking proved that BHs are indeed grey because of the thermal radiation emitted by the BHs due to the relativistic quantum effects. These radiation are known as Hawking radiation named after British Physicist Stephen Hawking [65]. In addition he anticipated that BH in the end can evaporate completely. As per Hawking's theory, BH isn't entirely "black" yet rather actually emits particles. These radiation, could loss the energy and mass of BH to make them escape. The emitted Hawking radiation are changed due the rotated continuum, while tending to structurally infinity. These discharged radiation are changed from at the geologically infinity radiation, the change can be indicated by greybody factor [66]-[74]. The greybody factor can be calculated by using different methods such as WKB approximation and matching technique [75]-[85].

In this paper we are focus to examine the impact of the nonlinear electrodynamics (NLED) at the bending angle where we utilize the GBT in which the bending of light becomes a global impact [86]. One of the most major issue from the beginning of the universe is singularity [87]-[92]. In GR, the spacetime singularities boost a whole lot of problems [93]-[99].

In classical general relativity, NLED has been proposed in order to eliminate spacetime singularities develop at the center of charged black holes [98]-[108]. Born and Infeld (BI) [109] gave the remarkable idea to remove singularities at the origin of point like charged particles, as a consequence, NLED provided a bounded electric field at the center of point-like charges. While in contrast to the NLED, linear electrodynamics provided an unbounded electric field, this implies the divergences in self-energy of point-like charges. Kruglov proposed one greater version of NLED with two parameters beta and gamma, where the particular extent of magnetic field, the unitary rules and causality are satisfied [94]. A lot of work has also been done on the consequences of NLED on GL [94]-[108]. In this paper, we worked for another model of NLED proposed by Mazharimousavi and Halilsoy [86], i.e., the static spherically symmetric electric and magnetic BH solutions. We examine the astrophysical phenomenon of bending of light for electric and magnetic BH solutions and examine the effects of NLED on GL.

This paper is organized as follows. In section 2, we give brief review about the solution of magnetic and electric BHs and find their optical geometry and then we discuss about GBT in detail and find the bending angle of BHs in non-plasma medium. In section 3, we discuss the bending angle using graphical analysis in detail in non-plasma medium. In the section $\mathbf{4}$, we obtain the bending angle in plasma medium for magnetic and electric BHs. In section $\mathbf{5}$, we discuss about the plots of the bending angle in plasma medium and after this in section $\mathbf{6}$, we calculate the bound of greybody factor for electric and magnetic BHs and then in the section 7, we look at the graphical behaviour of greybody bound. Section $\mathbf{8}$ is devoted to conclude the discussion about the results obtained from our analysis. alysis.

\section{WEAK GRAVITATIONAL LENSING IN NON-PLASMA MEDIUM FOR ELECTRIC AND MAGNETIC BLACK HOLES}

The action of the Einstein NLED theory has the form [86]

$$
S=\int d^{4} x \sqrt{-g}\left(\frac{1}{2 \kappa^{2}} \mathcal{R}+\mathcal{L}(\mathcal{F})\right),
$$

where $\mathcal{R}$ is the Ricci scalar and $\kappa^{2}=8 \pi G$, where $G$ is the Newton's constant in four dimensional spacetime and $\mathcal{L}=$ $-\frac{1}{\beta} \ln \left(\cos ^{2}(\sqrt{-\beta \mathcal{F}})\right)$, where $\beta$ is a constant with dimension $(\text { length })^{4}, \mathcal{F}=\frac{1}{4} F_{\mu \nu} F^{\mu \nu}$ and $F_{\nu \mu}=\partial_{\mu} A_{\nu}-\partial_{\nu} A_{\mu}$, Eq. $(1)$ implies the Einstein field equations as

$$
\mathcal{R}_{i j}-\frac{1}{2} g_{i j} \mathcal{R}=-\kappa^{2} T_{i j}
$$

where $\mathcal{R}_{i j}$ is the Ricci tensor. By varying Eq.(1), we determine the equation of motion for electromagnetic field

$$
\partial_{c}\left(\sqrt{-g}\left(F^{i j} \mathcal{L}_{F}+\tilde{F}^{i j} \mathcal{L}_{F}\right)\right)=0
$$

where, $\mathcal{L}_{F}=\frac{\partial \mathcal{L}}{\partial \mathcal{F}}=-\frac{\tan \sqrt{-\beta \mathcal{F}}}{\sqrt{-\beta \mathcal{F}}}$. In our analysis, we consider the static electric and magnetic BH solutions by applying equation of motion for electromagnetic field and Einstein field equations. Kruglov has observed magnetically charged BH in the context of NLED [94], while electrically charged BH solution is observed by Mazharimousavi and Halilsoy [93], and to solve the corresponding Einstein nonlinear Maxwell equations, the invariant is $\left(\mathcal{F}=-\frac{E^{2}}{2}=-\frac{q^{2}}{2 r^{4}}\right)$, where $q$ is the electric charge and for magnetic BH the invariant is $\left(\mathcal{F}=\frac{B^{2}}{2}=-\frac{p^{2}}{2 r^{4}}\right)$, where $p$ is the magnetic charge. Mazharimousavi and Halilsoy in their new model [86] have examined the static electric and magnetic solutions separately. 


\section{A. Optical Metric for Electric Black Hole}

The line-element for the electric and magnetic BHs in a static and spherically symmetric spacetime is defined by [86]

$$
d s^{2}=-f(r) d t^{2}+\frac{d r^{2}}{f(r)}+r^{2} d \Omega^{2}, \quad d \Omega^{2}=d \theta^{2}+\sin ^{2} \theta d \varphi^{2}
$$

where the metric function $f(r)$ for electric $\mathrm{BH}$ is defined as

$$
f(r)=1-\frac{2 G M}{r}-\frac{\pi q^{2} G}{2 r_{o}^{2}}+\mathcal{O}\left(r^{2} \ln r\right) \quad r \rightarrow 0,
$$

where $q$ is the electric charge of a $\mathrm{BH}, M$ is the real ADM mass of the $\mathrm{BH}$ and $r_{o}$ is the parameter. Supposing the spectator and source both are in a tropical plane and additionally, null photon with the $\left(\theta=\frac{\pi}{2}\right)$ inside the similar plane, we get optical space as

$$
d s^{2}=-f(r) d t^{2}+\frac{d r^{2}}{f(r)}+r^{2} d \varphi^{2} .
$$

For equatorial plane and the null geodesics $(d s=0)$, we obtain

$$
d t^{2}=\frac{d r^{2}}{(f(r))^{2}}+\frac{r^{2} d \varphi^{2}}{f(r)} .
$$

The non-zero Christoffel symbols of Eq.(7) are calculated as

$$
\begin{aligned}
& \Gamma_{00}^{0}=-\frac{f^{\prime}(r)}{f(r)}, \\
& \Gamma_{10}^{1}=\frac{1}{r}-\frac{f^{\prime}(r)}{2 f(r)}, \\
& \Gamma_{11}^{0}=\frac{-2 f(r)}{r}+f^{\prime}(r),
\end{aligned}
$$

in which 0 and 1 indicate $r$-coordinate and $\varphi$-coordinate and the Ricci scalar of the optical metric is computed as

$$
\mathcal{R}=f(r) f^{\prime \prime}(r)-\frac{\left(f^{\prime}(r)\right)^{2}}{2}
$$

Hence, the Gaussian optical curvature is evaluated as

$$
\mathcal{K}=\frac{\mathcal{R}}{2}
$$

The Gaussian optical curvature of electric $\mathrm{BH}$ in leading order term after calculating and putting the value of $\mathcal{R}$ in Eq.(10) is calculated as

$$
\mathcal{K} \simeq-\frac{2 G M}{r^{3}}+\frac{G^{2} M \pi q^{2}}{r_{o}^{2} r^{3}}+\mathcal{O}\left(M^{2}, q^{4}\right)
$$

\section{B. Bending Angle for Electric Black Hole}

Here, we use GBT to obtain the bending angle of electric BH, via GBT to the domain $\mathcal{M}_{C}$, given as [21]:

$$
\iint_{\mathcal{M}_{C}} \mathcal{K} d S+\oint_{\partial \mathcal{M}_{C}} k d t+\sum_{l} \epsilon_{l}=2 \pi \mathcal{X}\left(\mathcal{M}_{C}\right)
$$


wherein, $k$ indicates the geodesic curvature, written as $k=\breve{g}\left(\nabla_{\dot{\zeta}} \dot{\zeta}, \ddot{\zeta}\right)$ so that the $\breve{g}(\dot{\zeta}, \dot{\zeta})=1, \ddot{\zeta}$ symbolizes the unit acceleration vector and external angle of $l^{\text {th }}$ vertex symbolizes as $\epsilon_{l}$. We get the jump angles equal to $\frac{\pi}{2}$, as $\mathcal{C}$ goes to infinity, hence we obtain $\epsilon_{l}+\epsilon_{l l} \rightarrow \pi$. Here, Euler characteristic $\mathcal{X}\left(\mathcal{M}_{C}\right)$ is 1 as the region $\mathcal{M}_{C}$ is non-singular. So

$$
\iint_{\mathcal{M}_{C}} \mathcal{K} d S+\oint_{\partial \mathcal{M}_{C}} k d t+\epsilon_{l}=2 \pi \mathcal{X}\left(\mathcal{M}_{C}\right)
$$

where, $\epsilon_{l}=\pi$ represents the entire jump angle and $\alpha_{\tilde{g}}$ is geodesic, as the Euler characteristic number is indicated by $\mathcal{X}$ is 1 . As $C \rightarrow \infty$, the most effective element is to be find as $k\left(F_{C}\right)=\left|\nabla_{\dot{F}_{C}} \dot{F}_{C}\right|$. The radial component for the sake of the geodesic curvature is defined as [21]:

$$
\left(\nabla_{\dot{F}_{C}} \dot{F}_{C}\right)^{r}=\dot{F}_{C}^{\varphi} \partial_{\varphi} \dot{F}_{C}^{r}+\Gamma_{\varphi \varphi}^{r}\left(\dot{F}_{C}^{\varphi}\right)^{2}
$$

For very large $\mathrm{C}, F_{C}:=r(\varphi)=C=$ const. Thus, Eq.(14) becomes $\left(\dot{F}_{C}^{\varphi}\right)^{2}=\frac{1}{(f(r))^{2}}$. Recalling $\Gamma_{\varphi \varphi}^{r}=\frac{-2 f(r)}{r}+f^{\prime}(r)$, yields that

$$
\left(\nabla_{\dot{F}_{C}^{r}} \dot{F}_{C}^{r}\right)^{r} \rightarrow \frac{-1}{C}
$$

This shows that geodesic curvature does not depend upon the topological defects so, $k\left(F_{C}\right) \rightarrow \frac{1}{C}$, but from the Eq.(7) we can write $d t=C d \varphi$. We can obtain this;

$$
k\left(F_{C}\right) d t=\frac{1}{C} C d \varphi
$$

By taking into consideration all above equations, we get

$$
\iint_{\mathcal{M}_{C}} \mathcal{K} d s+\oint_{\partial \mathcal{M}_{C}} k d t \stackrel{R \rightarrow \infty}{=} \iint_{W_{\infty}} \mathcal{K} d S+\int_{0}^{\pi+\alpha} d \varphi .
$$

Supposing that at zeroth order in the weak bending domain the light ray obeys the straight-line approximation which is defined as $r=\frac{b}{\sin \varphi}$. Then, the bending angle can be obtained by using the Eq.(12) and (17) as follows

$$
\alpha=-\int_{0}^{\pi} \int_{\frac{b}{\sin \varphi}}^{\infty} \mathcal{K} \sqrt{\operatorname{det} \breve{g}} d r d \varphi
$$

where

$$
\sqrt{\operatorname{det\breve {g}}}=r\left(1+\frac{3 G M}{r}+\frac{3 q^{2} \pi G}{4 r_{o}^{2}}\right) .
$$

After putting the Gaussian curvature obtained in Eq.(11) upto the leading terms and Eq.(19) into Eq.(18), the bending angle for electric $\mathrm{BH}$ is computed as

$$
\alpha \simeq \frac{4 G M}{b}+\frac{G^{2} M \pi q^{2}}{r_{o}^{2} b}+\mathcal{O}\left(M^{2}, q^{4}\right) .
$$

The obtained bending angle (20) depends on the electric charge $q$, mass of the BH, parameter $r_{o}$ and impact parameter $b$. It is to be mention here that the bending angle $\alpha$ of electric BH in non-plasma medium reduces to Schwarzschild $\mathrm{BH}$ bending angle in non-plasma medium upto the order one of $M$ if we neglect the electric charge $q$.

\section{Optical Metric for Magnetic Black Hole}

In order to compute the Gaussian optical curvature for magnetic $\mathrm{BH}$, the spherically symmetric metric for the magnetic $\mathrm{BH}$ is defined by Eq.(4), where the metric function for the magnetic $\mathrm{BH}$ [86] is given as follows

$$
f(r)=1-\frac{2 G M}{r}+\frac{G p^{2}}{r^{2}}-\beta \frac{G p^{4}}{60 r^{6}}+\mathcal{O}\left(r^{-10}\right) \quad r \rightarrow \infty,
$$


where $p$ is the magnetic charge and $\beta$ is the parameter. After applying the basic procedure, the optical space in equatorial plane $\left(\theta=\frac{\pi}{2}\right)$ and for the null geodesic $\left(d s^{2}=0\right)$, we get the optical metric for the magnetic $\mathrm{BH}$ as follows:

$$
d t^{2}=\frac{d r^{2}}{\left(1-\frac{2 G M}{r}+\frac{G p^{2}}{r^{2}}-\beta \frac{G p^{4}}{60 r^{6}}\right)^{2}}+\frac{r^{2} d \varphi^{2}}{\left(1-\frac{2 G M}{r}+\frac{G p^{2}}{r^{2}}-\beta \frac{G p^{4}}{60 r^{6}}\right)} .
$$

The Gaussian optical curvature is defined in Eq.(10), by computing the Ricci scalar of the optical metric Eq.(22), the Gaussian optical curvature upto the leading order term of magnetize $\mathrm{BH}$ is calculated as

$$
\mathcal{K} \simeq-\frac{2 G M}{r^{3}}+\frac{3 G p^{2}}{r^{4}}-\beta \frac{7 G p^{4}}{20 r^{8}}+\mathcal{O}\left(G^{2}, M^{2}\right)
$$

\section{Bending Angle for Magnetic Black Hole}

In this segment, we are interesting in calculating the bending angle for magnetic BH by using GBT in non-plasma medium just as we calculated for electric BH in section (2.2). For obtaining the angle in weak bending limit, as the shaft of light chases the estimation for a straight line so by utilizing $r(t)=b / \sin \varphi$ at zeroth order

$$
\alpha=-\int_{0}^{\pi} \int_{b / \sin \varphi}^{\infty} \mathcal{K} \sqrt{\operatorname{det} \breve{g}} d r d \varphi
$$

where

$$
\sqrt{\operatorname{det} \breve{g}} d v=r\left(1+\frac{3 G M}{r}-\frac{3 p^{2} G}{2 r^{2}}+\beta \frac{G p^{4}}{40 r^{6}}\right) d r .
$$

By putting the value of $\mathcal{K}$ obtained in Eq.(23) and Eq.(25) in Eq.(24), the bending angle in non-plasma medium for magnetic $\mathrm{BH}$ is calculated as

$$
\alpha \simeq \frac{4 G M}{b}-\frac{3 G p^{2} \pi}{4 b^{2}}+\beta \frac{7 G p^{4} \pi}{384 b^{6}}+\mathcal{O}\left(G^{2}, M^{2}\right) .
$$

The obtained bending angle (26) depends on the magnetic charge $p$, mass of the black hole, parameter $\beta$ and impact parameter $b$. We also observe that the bending angle $\alpha$ of magnetic BH in non-plasma medium reduces to Schwarzschild BH bending angle in non-plasma medium upto the order one of $M$ if we neglect the magnetic charge $p$.

\section{GRAPHICAL ANALYSIS OF BENDING ANGLES IN NON-PLASMA MEDIUM}

This section is devoted to the study of the behaviour of bending angles of electric and magnetic BHs in non-plasma medium graphically and to explain the effect of electric charge $q$, impact parameter $b$ and parameter $r_{o}$ (in case of electric $\mathrm{BH}$ ) and magnetic charge $p$, impact parameter $b$ and the parameter $\beta$ (in case of magnetic $\mathrm{BH}$ ) on the deflection angles.

\section{A. For Electric Black Hole}

(i) $\mathrm{G}=\mathrm{M}=1, r_{0}=2$

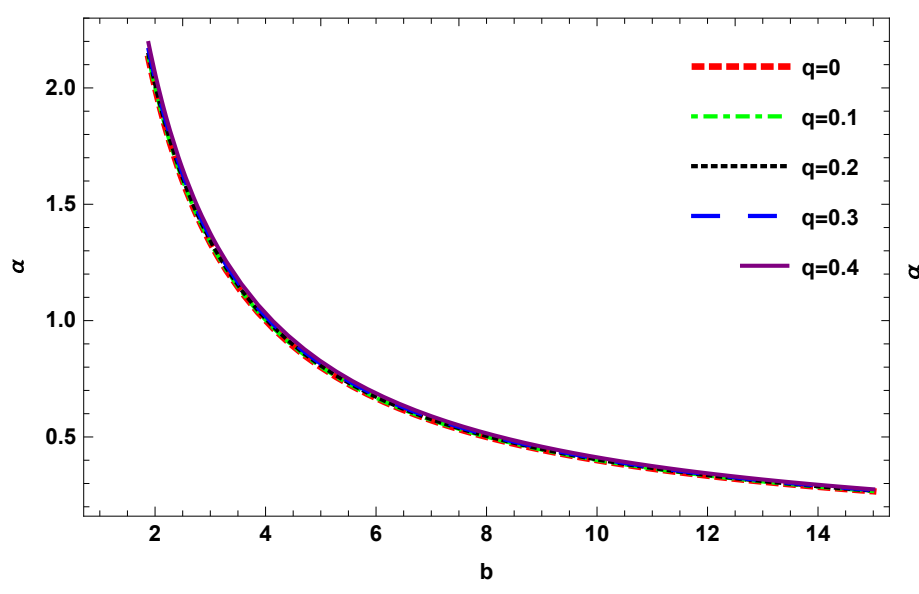

(ii) $\mathrm{G}=\mathrm{M}=1, r_{\mathrm{o}}=2$

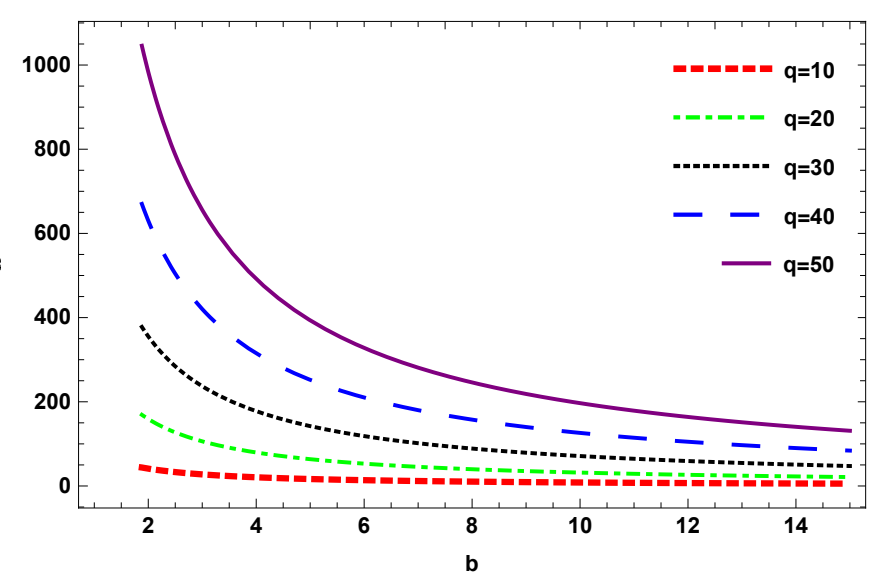


Figure 1: $\alpha$ versus $b$.

- Figure 1 demonstrates the graphical behaviour of the bending angle $\alpha$ of electric BH with respect to the impact parameter $b$ for the fixed value of $M, G$ and $r_{o}$.

(i) For the small values of $q$ graph between the the bending angle $\alpha$ and the impact parameter $b$ shows that the behaviour of the bending angle $\alpha$ is uniformly constant.

(ii) For the large values of $q$ graph between the the bending angle $\alpha$ and the impact parameter $b$ depicts that the behaviour of the bending angle $\alpha$ is increasing gradually.

(i) $\mathrm{G}=\mathrm{M}=1, r_{0}=2$

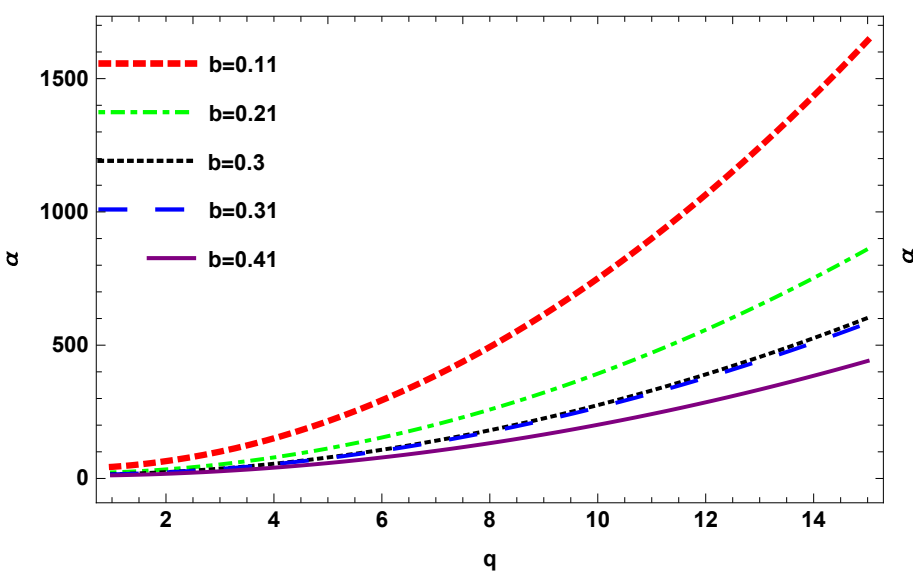

(ii) $\mathrm{G}=\mathrm{M}=1, r_{0}=2$

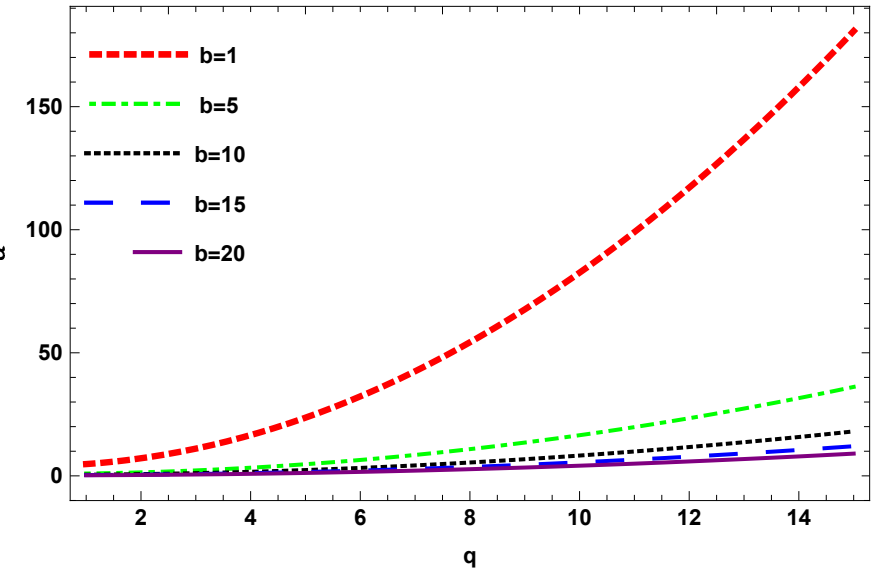

Figure 2: $\alpha$ versus $q$.

- Figure 2 exhibits the graphical behaviour of the bending angle $\alpha$ of electric BH with respect to the electric charge $q$ by setting $M, G$ and $r_{o}$ fixed.

(i) For the small values of impact parameter $b$ the graph of bending angle $\alpha$ with respect to electric charge $q$ exhibits that bending angle $\alpha$ shows decreasing behaviour.

(ii) For the large values of impact parameter $b$ the graph of bending angle $\alpha$ with respect to electric charge $q$ shows that for the values of $1<b<5$ bending angle $\alpha$ increasing but as the value of $b$ increasing the behaviour of the bending angle starts decreasing.

\section{B. For Magnetic Black Hole}

(i) $G=M=1, \beta=5$

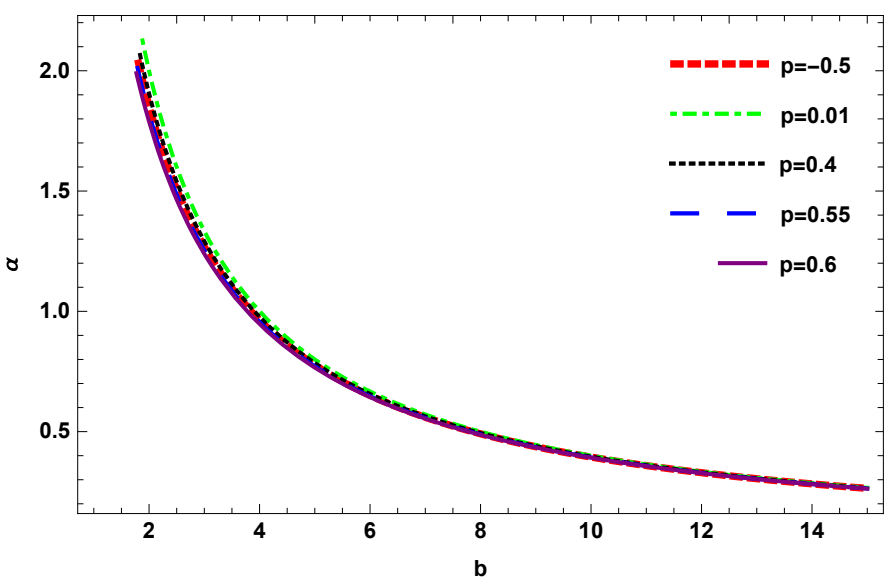

(ii) $\mathrm{G}=\mathrm{M}=1, \beta=10$

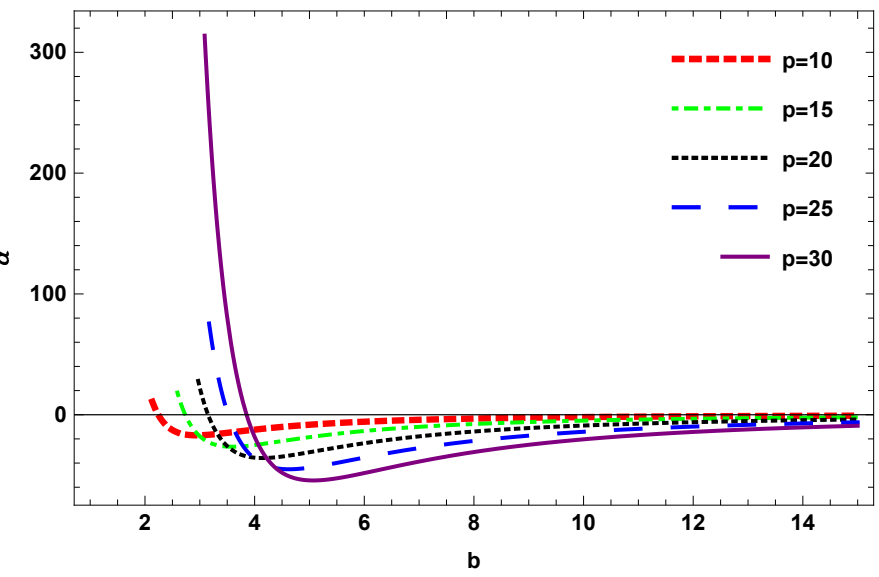

Figure 3: $\alpha$ versus $b$. 
- Figure 3 exhibits the graphical behaviour of bending angle $\alpha$ of magnetic BH with respect to the impact parameter $b$ for the fixed value of $M, G$ and $\beta$.

(i) Graph between bending angle $\alpha$ and impact parameter $b$ shows that for the very small variation of magnetic charge $p$ and parameter $\beta=5$ the bending angle $\alpha$ rapidly decreases.

(ii) Graph between bending angle $\alpha$ and impact parameter $b$ depicts that for the large value of magnetic charge $p$ and parameter $\beta=10$, the bending angle $\alpha$ increasing and then goes to infinity.

(i) $\mathrm{G}=\mathrm{M}=1, \mathrm{p}=\mathbf{0 . 4 5}$

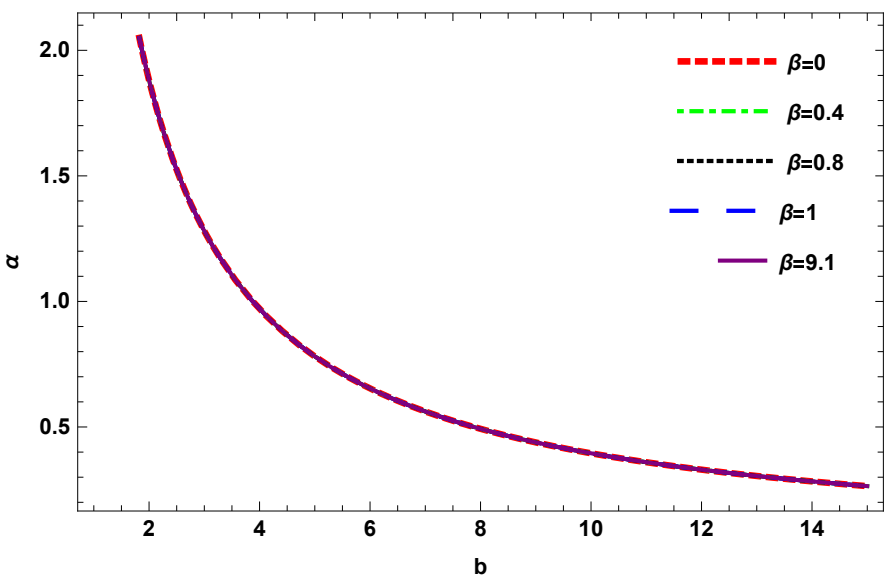

(ii) $\mathrm{G}=\mathrm{M}=1, \mathrm{p}=10$

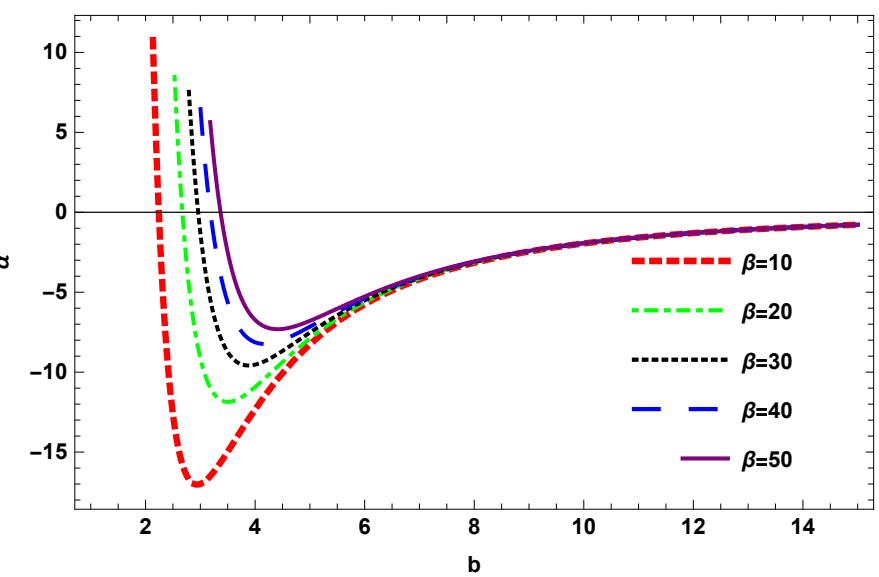

Figure 4: $\alpha$ versus $b$.

- Figure 4 represents the graphical behaviour of bending angle $\alpha$ of magnetic BH with respect to the impact parameter $b$ for the fixed value of magnetic charge $p, G$ and $M$.

(i) Graph between the bending angle $\alpha$ and impact parameter $b$ exhibits that for small values of parameter $\beta$ and magnetic charge $p=0.45$ the bending angle $\alpha$ decreasing constantly.

(ii) Graph between the bending angle $\alpha$ and impact parameter $b$ exhibits that for the large values of parameter $\beta$ and magnetic charge $p=10$ the bending angle $\alpha$ firstly starts decreasing negatively and then increasing and goes to the infinity.
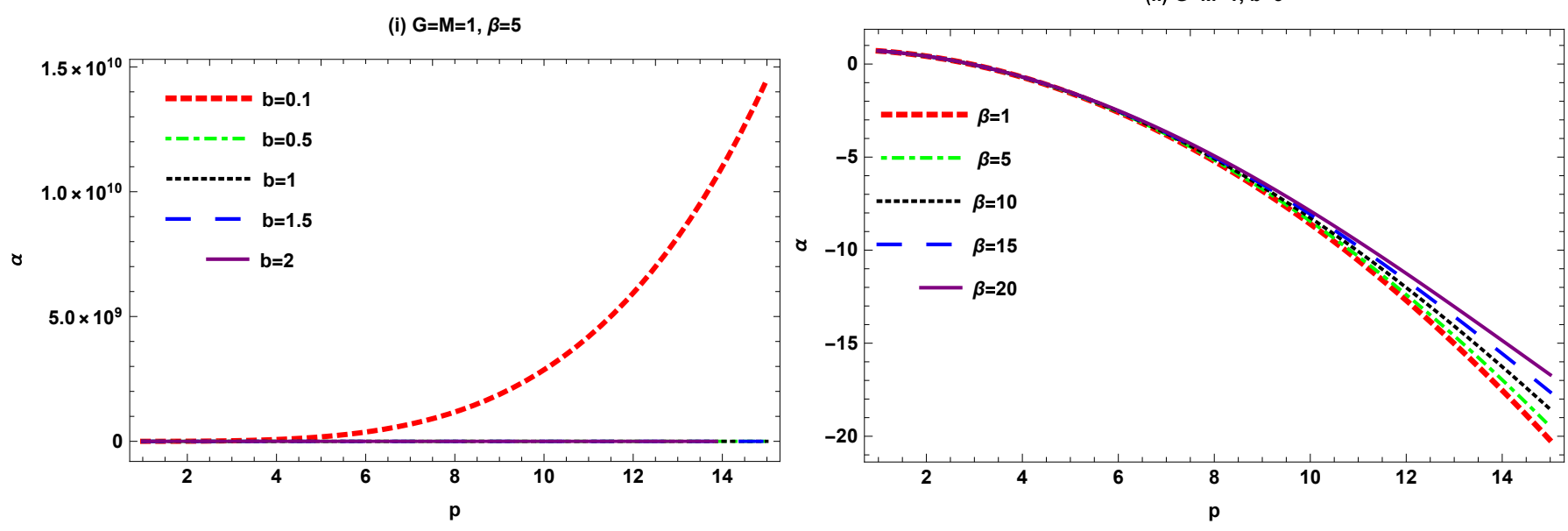

Figure 5: $\alpha$ versus $p$.

- Figure 5 demonstrates the graphical behaviour of bending angle $\alpha$ of magnetic $\mathrm{BH}$ with respect to the magnetic charge $p$ for the fixed value of $G$ and $M$.

(i) Graph between the bending angle $\alpha$ and magnetic charge $p$ shows that for the variation of impact parameter $b$ and parameter $\beta=5$ firstly the bending angle $\alpha$ increases then start decreasing. 
(ii) Graph between the bending angle $\alpha$ and magnetic charge $p$ for the variation in the value of parameter $\beta$ and impact parameter $b=5$ shows that the bending angle $\alpha$ is negatively increasing.
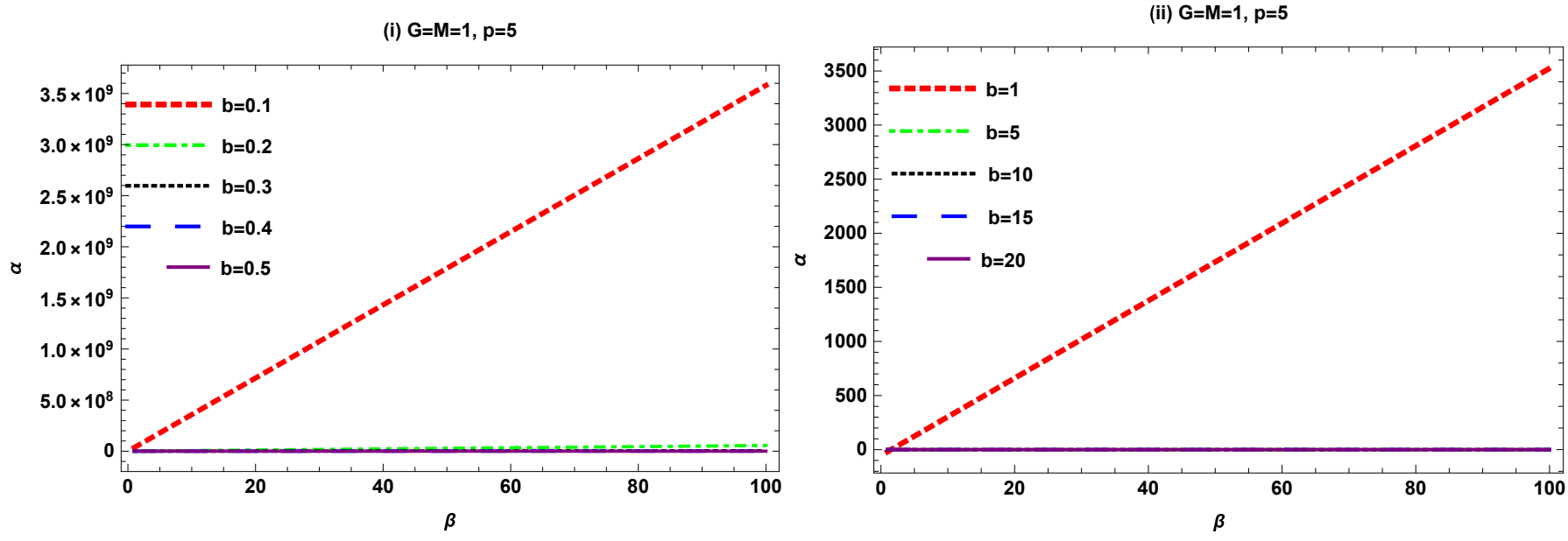

Figure 6: $\alpha$ versus $\beta$.

- Figure 6 represents the graphical behaviour of bending angle $\alpha$ of magnetic BH with respect to the $\beta$ and keeping magnetic charge $p, G$ and $M$ fixed.

(i) Graph between the bending angle $\alpha$ and $\beta$ exhibits that for the small variation of the impact parameter $b$ and magnetic charge $p=5$ the bending angle $\alpha$ is decreasing.

(ii) Graph between the bending angle $\alpha$ and $\beta$ for the large value of impact parameter $b$ and magnetic charge $p=5$ the bending angle $\alpha$ is decreasing.

\section{INFLUENCE OF PLASMA MEDIUM ON WEAK LENSING}

This section is devoted to the study of the impact of plasma medium on the weak lensing. The refractive index for the electric and magnetic BHs is given as [23]

$$
\dot{n^{2}}(r, \omega(r))=1-\frac{\omega_{e}^{2}(r)}{\omega_{\infty}^{2}(r)} .
$$

\section{A. Optical Metric and Bending Angle for Electric Black Hole}

The refractive index for the case of electric $\mathrm{BH}$ is given by

$$
n(r)=\sqrt{1-\frac{\omega_{e}^{2}}{\omega_{\infty}^{2}}\left(1-\frac{2 G M}{r}-\frac{\pi q^{2} G}{2 r_{o}^{2}}\right)},
$$

where $\omega_{e}$ indicates the electron plasma frequency and $\omega_{\infty}$ indicates light frequency computed by the viewer at infinity. The static spherically symmetric metric and the metric function is define as

$$
d s^{2}=-f(r) d t^{2}+\frac{d r^{2}}{f(r)}+r^{2}\left(d \theta^{2}+\sin ^{2} \theta d \varphi^{2}\right)
$$

and

$$
f(r)=1-\frac{2 G M}{r}-\frac{\pi q^{2} G}{2 r_{o}^{2}} .
$$

To get optical measurement, by considering light source and the spectator likewise null photon are in the tropical plane with $\left(\theta=\frac{\pi}{2}\right)$. Now, for null geodesic put $d s^{2}=0$, we get

$$
d t^{2}=g_{m w}^{o p t} d x^{m} d x^{w}=n^{2}\left[\frac{d r^{2}}{f^{2}(r)}+\frac{r^{2} d \varphi^{2}}{f(r)}\right]
$$


with determinant $g_{m w}^{o p t}$,

$$
\sqrt{g^{o p t}}=r\left(1-\frac{\omega_{e}^{2}}{\omega_{\infty}^{2}}\right)+G M\left(3-\frac{\omega_{e}^{2}}{\omega_{\infty}^{2}}\right)+\frac{\pi q^{2} G r}{4 r_{o}^{2}}\left(3-\frac{\omega_{e}^{2}}{\omega_{\infty}^{2}}\right) .
$$

The non-zero Christoffel Symbols by using Eq.(31) are calculated as

$$
\begin{aligned}
& \Gamma_{00}^{0}=\frac{\left(f(r) \omega_{e}^{2}-2 \omega_{\infty}^{2}\right)\left(f(r) \omega_{e}^{2}+\omega_{\infty}^{2}\right) f^{\prime}(r)}{2 f(r) \omega_{\infty}^{4}}, \\
& \Gamma_{10}^{1}=\frac{r}{2}\left(\frac{(f(r))^{3} \omega_{e}^{4}}{\omega_{\infty}^{4}}+r f^{\prime}(r)-2 f(r)+\frac{r \omega_{e}^{2} f^{\prime}(r)}{\omega_{\infty}^{2}} f(r)\right), \\
& \Gamma_{11}^{0}=\frac{1}{r}-\frac{(f(r))^{2} \omega_{e}^{4}}{r \omega_{\infty}^{4}}-\frac{f^{\prime}(r)}{2 f(r)},
\end{aligned}
$$

where 0 and 1 indicate $r$-coordinate and $\varphi$-coordinate. The $\mathcal{K}$ can be obtained by utilizing the above Christoffel symbols as

$$
\mathcal{K}=\frac{R_{r \varphi r \varphi}\left(g^{o p t}\right)}{\operatorname{det}\left(g^{o p t}\right)}
$$

with the help of Eq.(33), the $\mathcal{K}$ for the electric $\mathrm{BH}$ is computed as

$$
\mathcal{K} \simeq-\frac{2 G M}{r^{3}}+\frac{G^{2} M \pi q^{2}}{r_{o}^{2} r^{3}}-\frac{3 G M \omega_{e}^{2}}{r^{3} \omega_{\infty}^{2}}+\frac{3 G^{2} M \pi q^{2} \omega_{e}^{2}}{r_{o}^{2} r^{3} \omega_{\infty}^{2}}+\mathcal{O}\left(M^{2}, q^{4}\right)
$$

Using GBT we find the bending angle for electric $\mathrm{BH}$ to relate it with non-plasma medium. To compute weak-field area, we apply the straight line approximation and that $r(t)=b / \sin \varphi$ at zeroth order

$$
\alpha=-\int_{0}^{\pi} \int_{b / \sin \varphi}^{\infty} \mathcal{K} d S
$$

Hence, the bending angle of light is calculated as

$$
\alpha \simeq \frac{4 G M}{b}+\frac{G^{2} M \pi q^{2}}{r_{o}^{2} b}+\frac{2 G M \omega_{e}^{2}}{b \omega_{\infty}^{2}}-\frac{G^{2} M \pi q^{2} \omega_{e}^{2}}{2 r_{o}^{2} b \omega_{\infty}^{2}}+\mathcal{O}\left(M^{2}, q^{4}\right) .
$$

The obtained bending angle (36) depends on the electric charge $q$, mass of the $\mathrm{BH}$, parameter $r_{o}$ and impact parameter $b$. We also observe that the bending angle $\alpha$ of electric $\mathrm{BH}$ in plasma medium reduces into Schwarzschild $\mathrm{BH}$ bending angle in plasma medium upto the order one of $M$ if we neglect the electric charge $q$. It is also to be mention here that the bending angle obtained in case of plasma medium (36) reduces to the bending angle obtained in the non-plasma medium (20) by neglecting the plasma terms.

\section{B. Optical Metric and Bending Angle for Magnetic Black Hole}

The refractive index in case of magnetic $\mathrm{BH}$ is

$$
n(r)=\sqrt{1-\frac{\omega_{e}^{2}}{\omega_{\infty}^{2}}\left(1-\frac{2 G M}{r}+\frac{p^{2} G}{r^{2}}-\beta \frac{G p^{4}}{60 r^{6}}\right)},
$$

where $\omega_{e}$ denotes electron plasma frequency and $\omega_{\infty}$ represents light frequency computed by the spectator at infinity. The corresponding metric in optical space characterized as

$$
d t^{2}=g_{l m}^{o p t} d x^{l} d x^{m}=n^{2}\left[\frac{d r^{2}}{f^{2}(r)}+\frac{r^{2} d \varphi^{2}}{f(r)}\right]
$$


The Gaussian curvature is obtained by using Christoffel symbols can be written as

$$
\mathcal{K}=\frac{R_{r \varphi r \varphi}\left(g^{o p t}\right)}{\operatorname{det}\left(g^{o p t}\right)}
$$

Using Eq.(39), the Gaussian curvature for the magnetic BH in case of plasma medium is determined as

$$
\mathcal{K} \simeq-\frac{2 G M}{r^{3}}+\frac{3 G p^{2}}{r^{4}}-\beta \frac{7 G p^{4}}{20 r^{8}}-\frac{3 G M \omega_{e}^{2}}{r^{3} \omega_{\infty}^{2}}+\frac{5 G p^{2} \omega_{e}^{2}}{r^{4} \omega_{\infty}^{2}}-\beta \frac{13 G p^{4} \omega_{e}^{2}}{20 r^{8} \omega_{\infty}^{2}}+\mathcal{O}\left(G^{2}, M^{2}\right)
$$

Using GBT we check the bending angle of magnetic BH to contrast it with non-plasma medium. Along these lines, for getting the point in the weak bending limit, as the light emission seeks after a straight line estimation so by using an instance of $r=b / \sin \varphi$ at zeroth order

$$
\alpha=-\int_{0}^{\pi} \int_{b / \sin \varphi}^{\infty} \mathcal{K} d S
$$

Using the value of $\mathcal{K}$ in (41) the bending angle for the magnetic $\mathrm{BH}$ in plasma medium is computed as

$$
\alpha \simeq \frac{4 G M}{b}-\frac{3 G p^{2} \pi}{4 b^{2}}+\beta \frac{7 G p^{4} \pi}{384 b^{6}}+\frac{2 G M \omega_{e}^{2}}{b \omega_{\infty}^{2}}-\frac{G p^{2} \pi \omega_{e}^{2}}{2 b^{2} \omega_{\infty}^{2}}+\beta \frac{G p^{4} \pi \omega_{e}^{2}}{64 b^{6} \omega_{\infty}^{2}}+\mathcal{O}\left(G^{2}, M^{2}\right) .
$$

The obtained bending angle (42) depends on the magnetic charge $p$, mass of the black hole, parameter $\beta$ and impact parameter $b$. We also observe that the bending angle $\alpha$ of magnetic $\mathrm{BH}$ in plasma medium reduces to Schwarzschild $\mathrm{BH}$ bending angle in plasma medium upto the order one of $M$ if we neglect the magnetic charge $q$. We also observe that the bending angle (42) obtained for magnetic BH in plasma medium reduces to the bending angle (26) obtained in case of non-plasma medium (26), when $\frac{\omega_{e}^{2}}{\omega_{\infty}^{2}} \rightarrow 0$.

\section{GRAPHICAL ANALYSIS OF BENDING ANGLES IN PLASMA MEDIUM}

This section is devoted to the study of the behaviour of bending angles of electric and magnetic BHs in plasma medium graphically and to explain the influence of plasma, electric charge $q$, impact parameter $b$ and parameter $r_{o}$ (in case of electric $\mathrm{BH}$ ) and magnetic charge $p$, impact parameter $b$ and the parameter $\beta$ (in case of magnetic $\mathrm{BH}$ ) on the deflection angles.

\section{A. For electric Black Hole}

(i) $\mathrm{G}=\mathrm{M}=1, r_{0}=2$

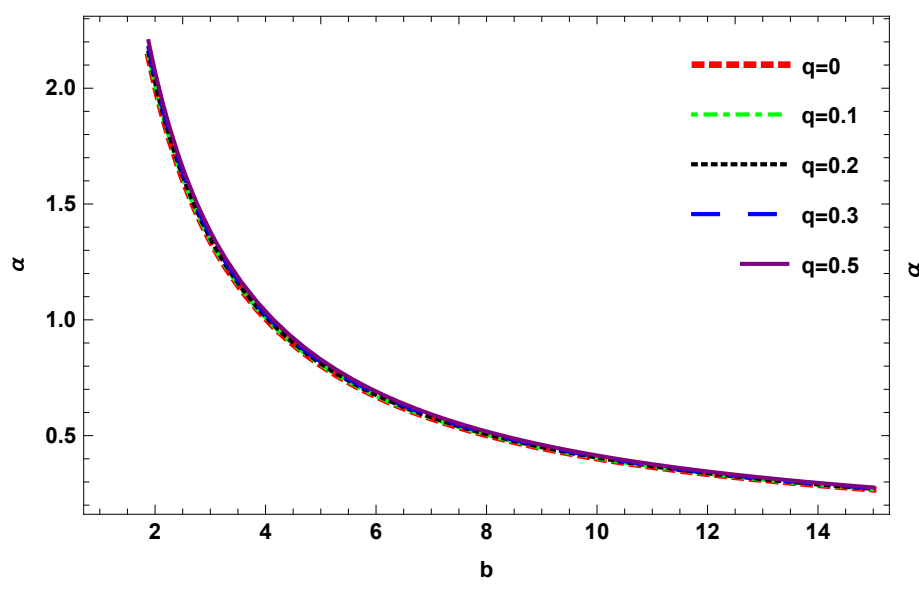

(ii) $\mathrm{G}=\mathrm{M}=1, r_{0}=2$

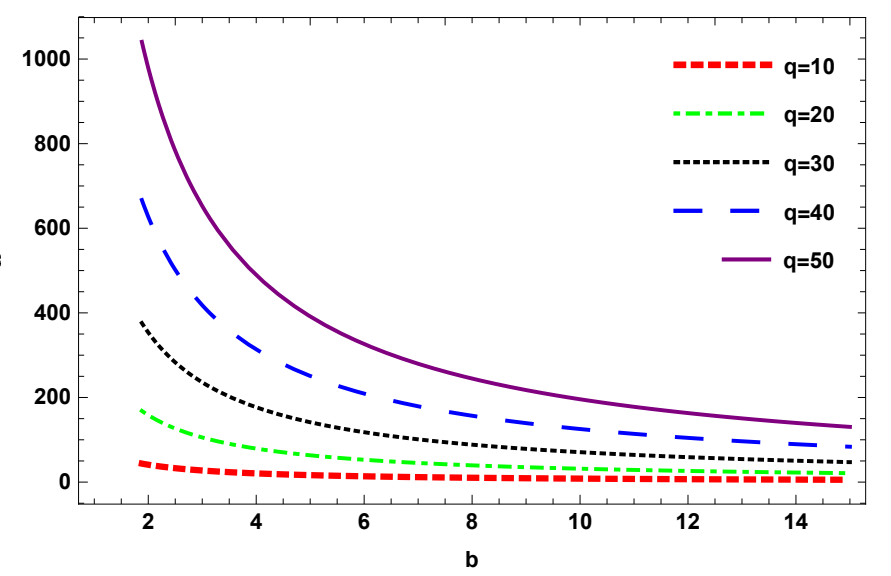

Figure 7: $\alpha$ versus $b$.

- Figure 7 demonstrates the graphical behaviour of the bending angle $\alpha$ of electric BH with respect to the impact parameter $b$ for $\frac{\omega_{e}^{2}}{\omega_{\infty}^{2}}=0.1$ and fixed values of $M, G, r_{o}$. 
(i) For the small values of $q$ graph between the the bending angle $\alpha$ and the impact parameter $b$ shows that the behaviour of the bending angle $\alpha$ is uniformly constant.

(ii) For the large values of $q$ graph between the the bending angle $\alpha$ and the impact parameter $b$ depicts that the behaviour of the bending angle $\alpha$ is increasing gradually.

(i) $\mathrm{G}=\mathrm{M}=1, r_{0}=2$

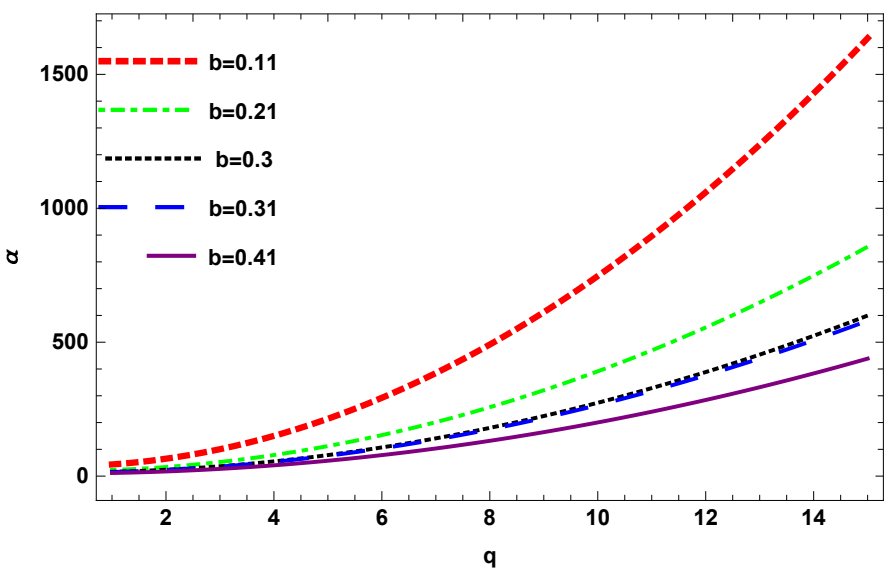

(ii) $\mathrm{G}=\mathrm{M}=1, r_{o}=2$

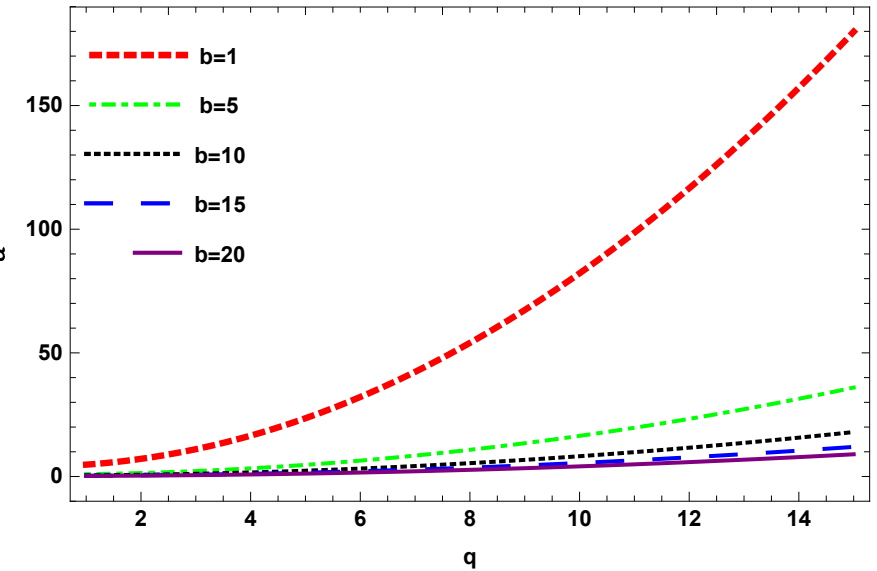

Figure 8: $\alpha$ versus $q$.

- Figure 8 demonstrates the graphical behaviour of bending angle $\alpha$ of magnetic $\mathrm{BH}$ with respect to the electric charge $q$ for $\frac{\omega_{e}^{2}}{\omega_{\infty}^{2}}=0.1$ and fixed values of $M, G, r_{o}$.

(i) For the small values of impact parameter $b$ the graph of bending angle $\alpha$ with respect to electric charge $q$ exhibits that the bending angle $\alpha$ shows decreasing behaviour.

(ii) For the large values of $b$ the graph of bending angle $\alpha$ with respect to electric charge $q$ shows that for the values of $1<b<5$ the bending angle $\alpha$ increasing but as the value of impact parameter $b$ increasing the bending angle $\alpha$ starts decreasing.

\section{B. For Magnetic Black Hole}

(i) $G=M=1, \beta=5$

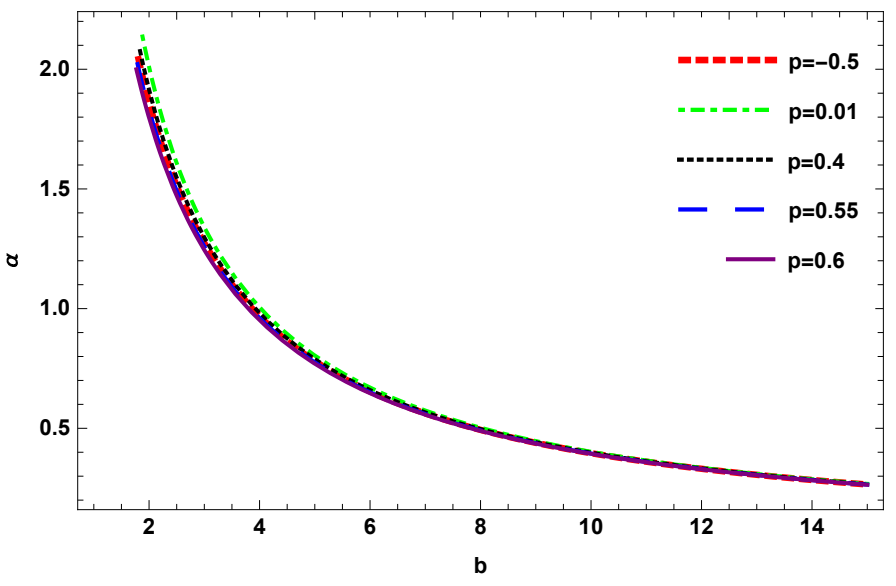

(ii) $\mathrm{G}=\mathrm{M}=1, \beta=10$

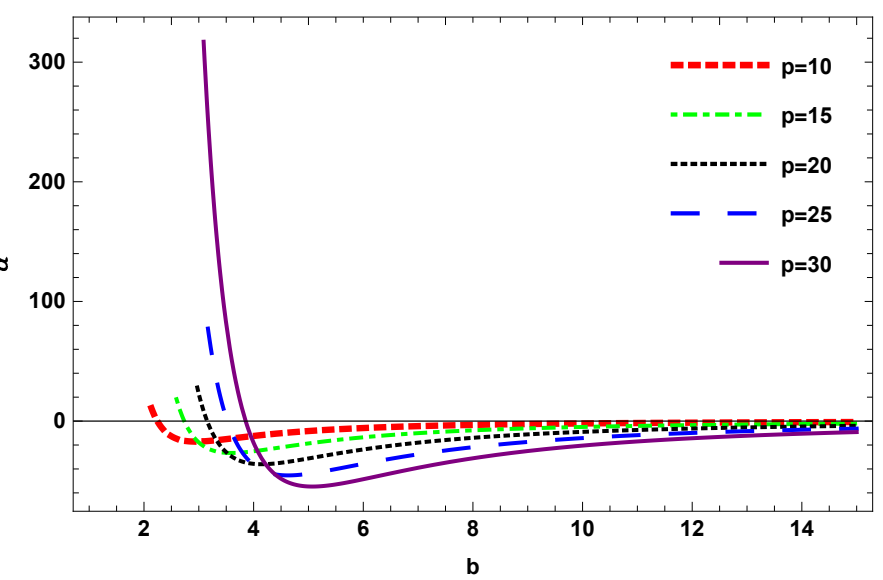

Figure 9: $\alpha$ versus $b$.

- Figure 9 exhibits the graphical behaviour of bending angle $\alpha$ of magnetic BH with respect to the impact parameter $b$ for $\frac{\omega_{e}^{2}}{\omega_{\infty}^{2}}=0.1$ and fixed values of $M, G, \beta$.

(i) Graph between bending angle $\alpha$ and impact parameter $b$ shows that for the very small variation of magnetic charge $p$ and parameter $\beta=5$ the bending angle $\alpha$ rapidly decreases. 
(ii) Graph between bending angle $\alpha$ and impact parameter $b$ depicts that for the large value of magnetic charge $p$ and parameter $\beta=10$, the bending angle $\alpha$ increasing and then goes to infinity.

(i) $\mathrm{G}=\mathrm{M}=1, \mathrm{p}=0.45$

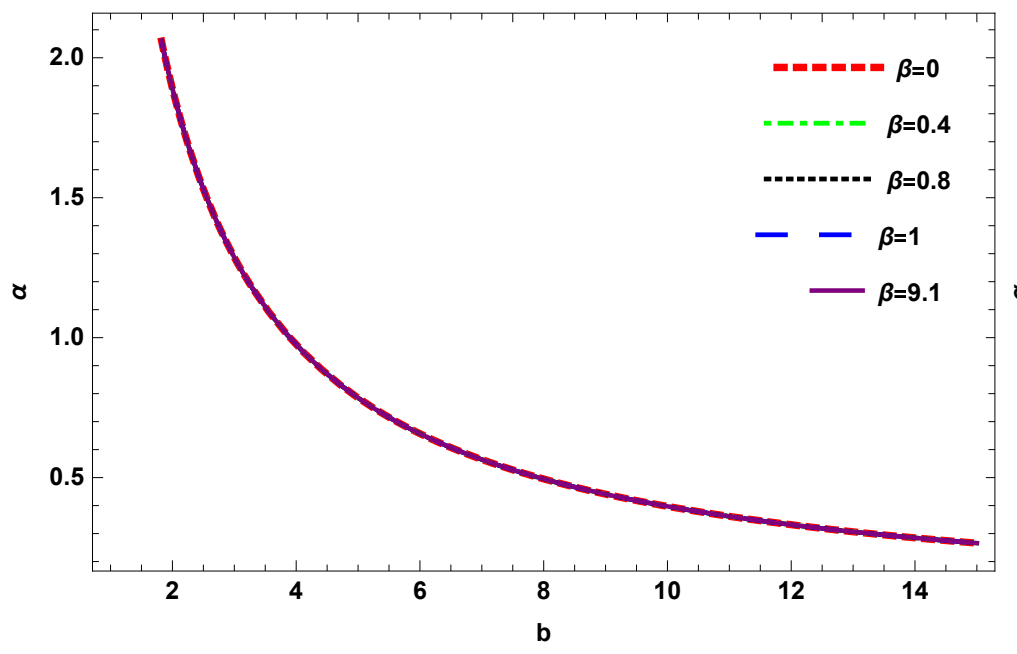

(ii) $\mathrm{G}=\mathrm{M}=1, \mathrm{p}=10$

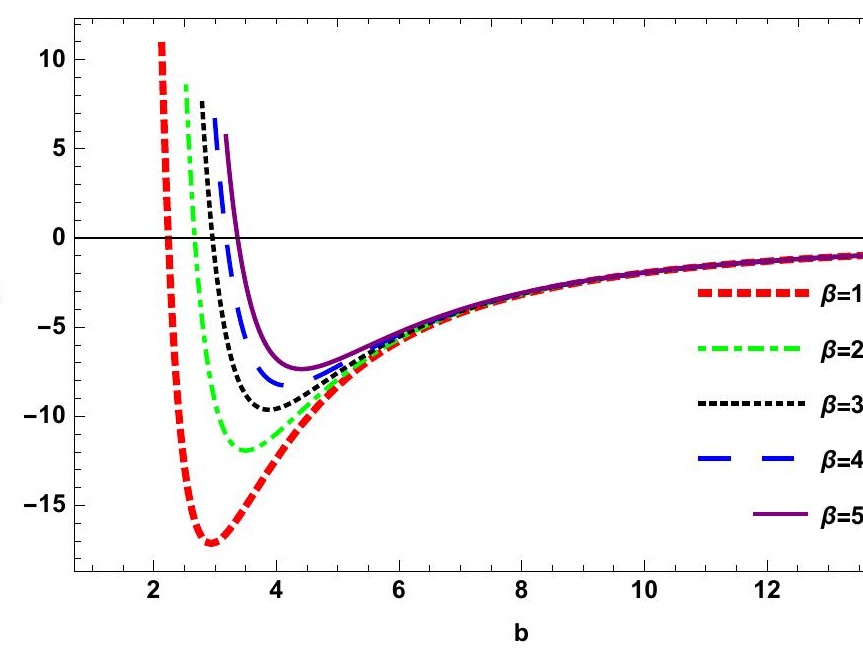

- Figure 10 represents the graphical behaviour of bending angle $\alpha$ of magnetic BH with respect to the impact parameter $b$ and for $\frac{\omega_{e}^{2}}{\omega_{\infty}^{2}}=0.1$ and fixed values of magnetic charge $p, G, M$.

(i) Graph between the bending angle $\alpha$ and impact parameter $b$ exhibits that for small values of parameter $\beta$ and magnetic charge $p=0.45$ the bending angle $\alpha$ is decreasing constantly.

(ii) Graph between the bending angle $\alpha$ and impact parameter $b$ exhibits that for the large values of parameter $\beta$ and magnetic charge $p=10$ the bending angle $\alpha$ firstly starts decreasing negatively and then increasing and goes to the infinity.

(i) $G=M=1, \beta=5$

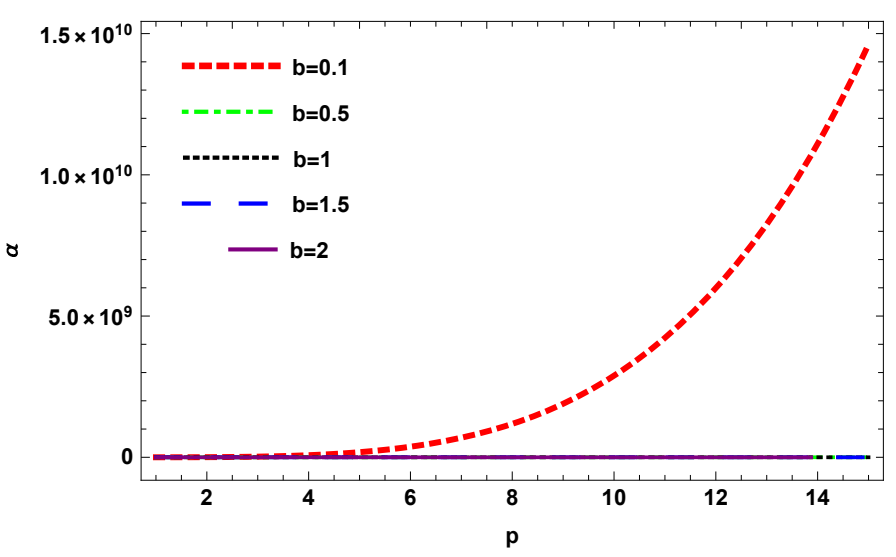

(ii) $\mathrm{G}=\mathrm{M}=1, \mathrm{~b}=5$

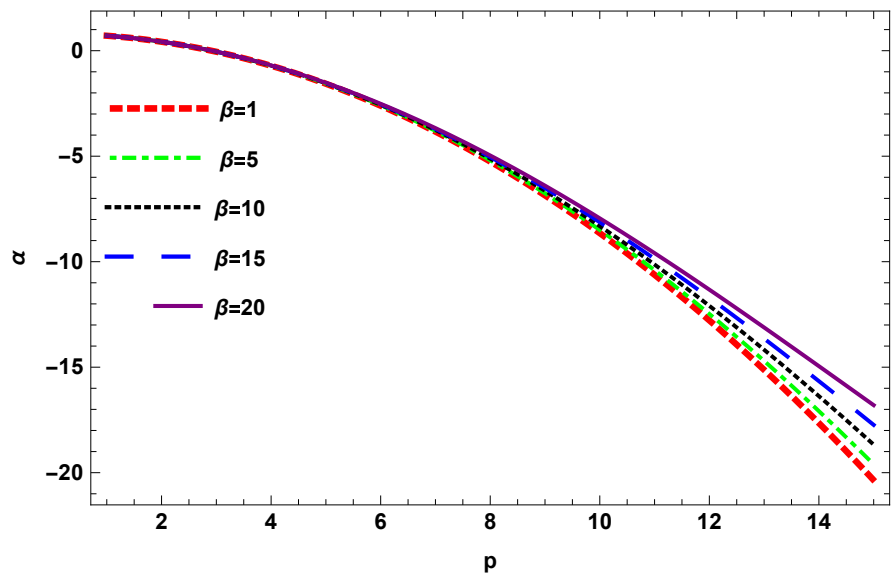

Figure 11: $\alpha$ versus $p$.

- Figure 11 demonstrates the graphical behaviour of bending angle $\alpha$ of magnetic $\mathrm{BH}$ with respect to the magnetic charge $p$ for $\frac{\omega_{e}^{2}}{\omega_{\infty}^{2}}=0.1$ and fixed values of $G, M$.

(i) Graph between the bending angle $\alpha$ and magnetic charge $p$ shows that for the variation of impact parameter $b$ and parameter $\beta=5$ firstly bending angle $\alpha$ increases then start decreasing.

(ii) Graph between the bending angle $\alpha$ and magnetic charge $p$ for the variation in the value of $\beta$ and impact parameter $b=5$ shows that the bending angle $\alpha$ negatively increasing. 
(i) $\mathrm{G}=\mathrm{M}=1, \mathrm{p}=5$

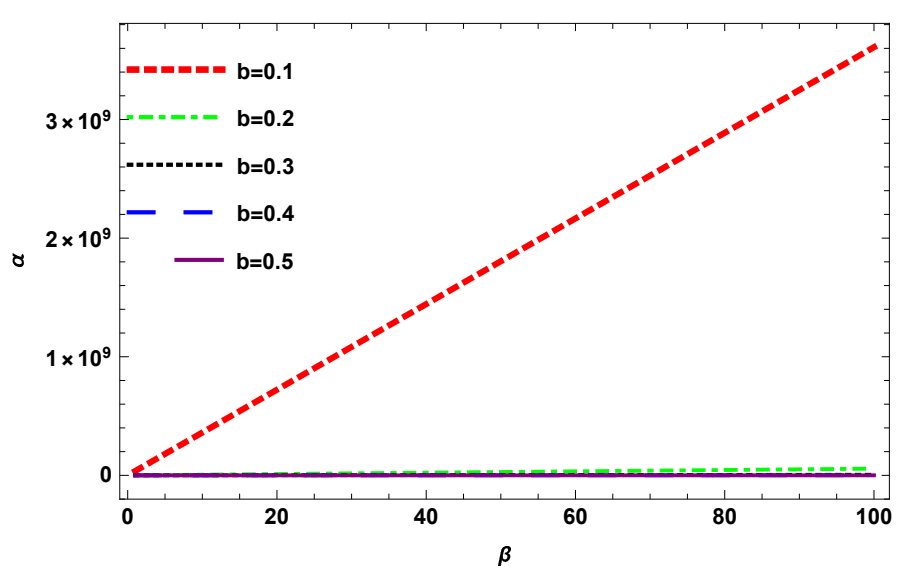

(ii) $\mathrm{G}=\mathrm{M}=1, \mathrm{p}=5$

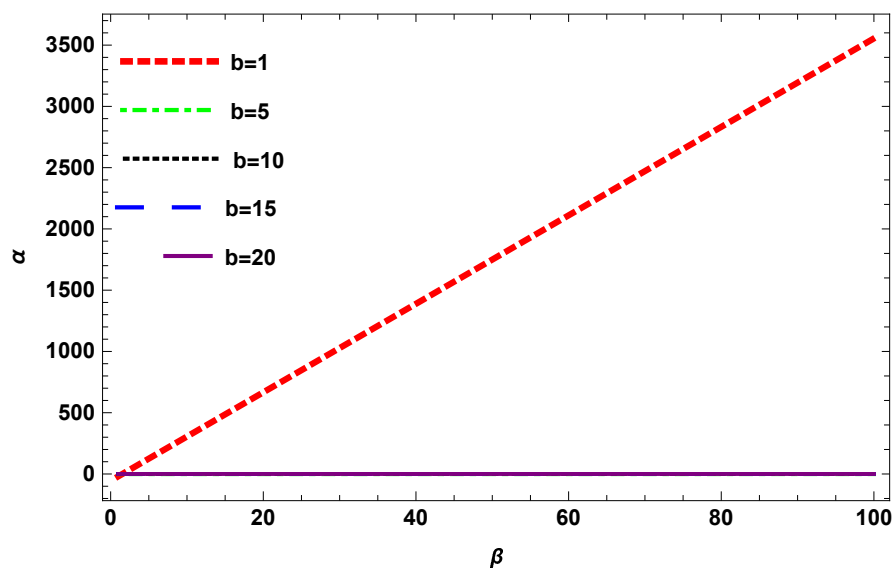

Figure 12: $\alpha$ versus $\beta$.

- Figure 12 represents the graphical behaviour of bending angle $\alpha$ of magnetic BH with respect to the $\beta$ for $\frac{\omega_{e}^{2}}{\omega_{\infty}^{2}}=0.1$ and fixed values of magnetic charge $p, G$ and $M$.

(i) Graph between the bending angle $\alpha$ and $\beta$ exhibits that for the small variation of the impact parameter $b$ and magnetic charge $p=5$ bending angle $\alpha$ is decreasing.

(ii) Graph between the bending angle $\alpha$ and $\beta$ for the large value of impact parameter $b$ and magnetic charge $p=5$ shows that the bending angle $\alpha$ is decreasing.

\section{GREYBODY FACTOR FOR ELECTRIC AND MAGNETIC BLACK HOLES}

In this part, we work out for the bound of greybody factor for the electric and magnetic BHs. There are numerous strategies to figure the greybody variable like matching teachnique and WKB approximation [76]. In this current work, we will consider the technique that doesn't utilize such approximations, for example, rigorous lower bound on the greybody factor [77]-[85].

\section{A. For Electric Black Hole} is

The spherically symmetric and static form of a line-element defined by Eq.(4), where the metric function for the electric BH

$$
f(r)=1-\frac{2 G M}{r}-\frac{\pi q^{2} G}{2 r_{o}^{2}}+\mathcal{O}\left(r^{2} \ln r\right) .
$$

The event horizon is obtained by taking $f(r)=0$, we get

$$
r_{h}=\frac{4 r_{o}^{2} G M}{2 r_{o}^{2}-G \pi q^{2}} .
$$

The Schrodinger-like equation is demonstrated as

$$
\left(\frac{d^{2}}{d r_{*}^{2}}+\omega^{2}-\check{\check{V}}(r)\right) \psi=0,
$$

where, $d r_{*}^{2}=\frac{1}{f(r)} d r$ and $r_{*}$ represents the "tortoise coordinate" and $\check{V}(r)$ indicates the potential which is given by

$$
\check{\check{V}}(r)=\frac{f(r) f^{\prime}(r)}{r}+l(l+1) \frac{f(r)}{r^{2}} .
$$

For $h=\omega$, the lower bound on the transmission probability is defined as [79]

$$
T \geq \frac{1}{\cosh ^{2}}\left(\frac{1}{2 \omega} \int_{r_{h}}^{\infty} \frac{\check{\tilde{V}}(r)}{f(r)} d r\right) .
$$


Using the value of $\check{V}(r)$ in Eq.(47) we get the following expressions

$$
\begin{aligned}
& =\frac{1}{\cosh ^{2}}\left[\frac{1}{2 \omega} \int_{r_{h}}^{\infty}\left(\frac{f^{\prime}(r)}{r}+\frac{l(l+1)}{r^{2}}\right) d r\right], \\
& =\frac{1}{\cosh ^{2}}\left[\frac{1}{2 \omega}\left(\frac{G M}{r_{h}^{2}}+\frac{l(l+1)}{r_{h}}\right)\right] .
\end{aligned}
$$

Substituting the value of $r_{h}$ in Eq.(49), we attain the bound as

$$
T \geq \frac{1}{\cosh ^{2}}\left[\frac{1}{2 \omega}\left(\frac{\left(G \pi q^{2}-2 r_{o}^{2}\right)^{2}}{16 r_{o}^{4} G M}-\frac{l(l+1)\left(G \pi q^{2}-2 r_{o}^{2}\right)}{4 r_{o}^{2} G M}\right)\right] .
$$

The above equation represents the lower bound for the greybody factor of electric charge $\mathrm{BH}$ which depends on the mass of the $\mathrm{BH}, r_{o}$ and the electric charge $q$ of the $\mathrm{BH}$. In the absence of the electric charge $q$, the above bound reduces to the form

$$
T \geq \frac{1}{\cosh ^{2}}\left[\frac{2 l(l+1)+1}{8 \omega G M}\right]
$$

which is the lower bound of the Schwarzschild BH [79].

\section{B. For Magnetic Black Hole}

The spherically symmetric form of a metric is defined in Eq.(4), where the metric function for the magnetic $\mathrm{BH}$ is [86]:

$$
f(r)=1-\frac{2 G M}{r}+\frac{p^{2} G}{r^{2}}-\beta \frac{G p^{4}}{60 r^{6}}+\mathcal{O}\left(r^{-10}\right) .
$$

The event horizon for the magnetic BH can not be calculated numerically, so in our analysis we calculated it graphically. For this we plot the graph between metric function $f(r)$ and $r$-coordinate by keeping parameter $\beta$ and magnetic charge $p$ fixed.

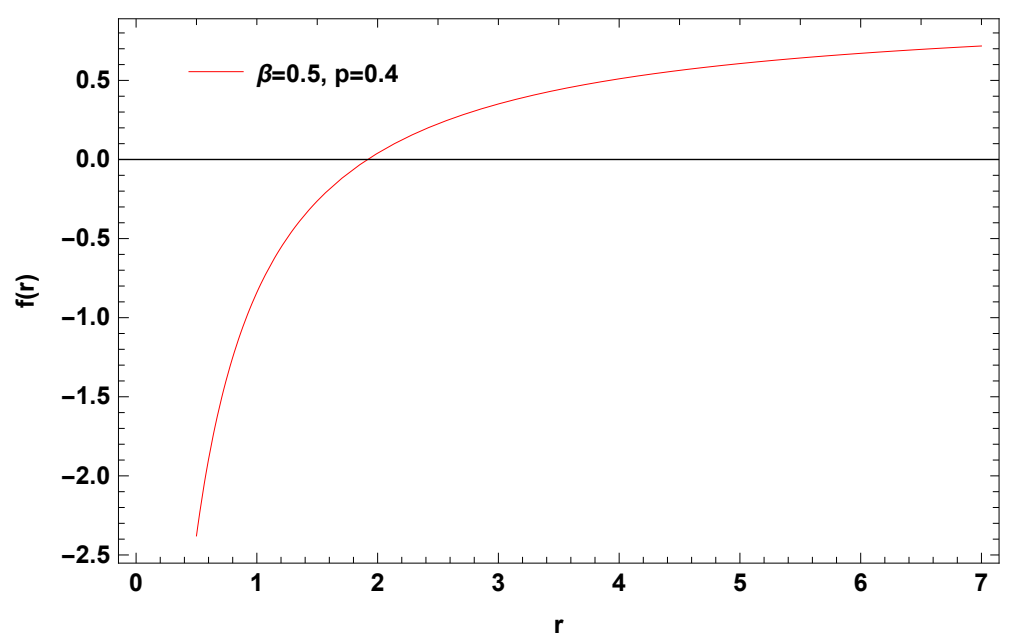

Figure 13: $f(r)$ versus $r$.

From figure 13, we get $r=1.8$ and the value of event horizon $r_{h}=\frac{1}{r}=0.555$. Now by using the Eq.(48), we get

$$
T \geq \frac{1}{\cosh ^{2}}\left[\frac{1}{2 \omega}\left(\frac{G M}{r_{h}^{2}}+\frac{l(l+1)}{r_{h}}-\frac{2 G p^{2}}{3 r_{h}^{3}}+\frac{G p^{4} \beta}{70 r_{h}^{7}}\right)\right] .
$$

By putting the value of $r_{h}$ in (53), we obtain the rigorous bound

$$
T \geq \frac{1}{\cosh ^{2}}\left[\frac{3.24649 G M-3.89969 G p^{2}+1.8018 l(l+1)+0.880747 G p^{4} \beta}{2 \omega}\right] .
$$

The above equation is the bound for the greybody factor of the magnetic $\mathrm{BH}$ which depend on the mass of the $\mathrm{BH}$, magnetic charge $p$ and parameter $\beta$. 


\section{GRAPHICAL ANALYSIS OF GREYBODY BOUND}

This section represents the graphical affects of greybody bound for electric and magnetic BHs and potential (we set $\check{V}(r)=$ $V(r)$ ) at the same time as for the distinct values of the charges of BHs with angular momentum $l=0,1$ respectively.

\section{A. For Electric Black Hole}

(i) $\mathrm{G}=\mathrm{M}=1, r_{0}=2$

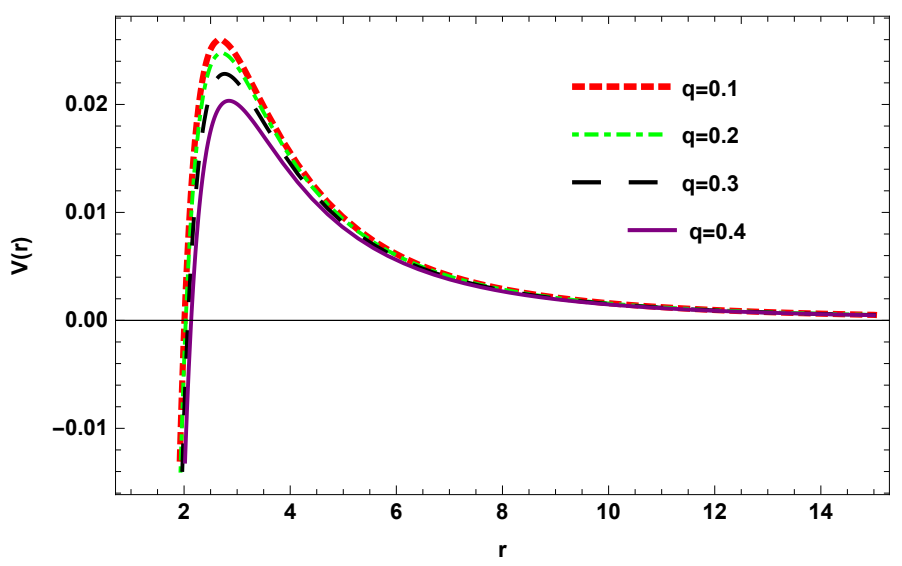

(ii) $\mathrm{G}=\mathrm{M}=1, r_{0}=2$

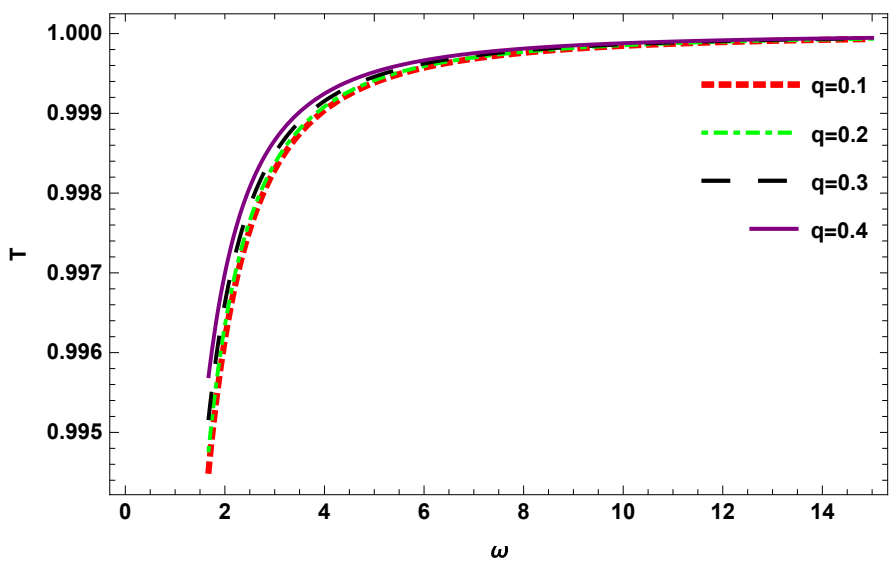

Figure 14: (i) indicates the potential with $l=0$ and (ii) indicates the corresponding greybody factor bound of electric $\mathrm{BH}$.

- We observe that value of the potential with $l=0$ increases when the value of the electric charge $q$ decreases and so the bound of the greybody factor becomes lower as it becomes difficult for the wave to transmit through the higher potential.
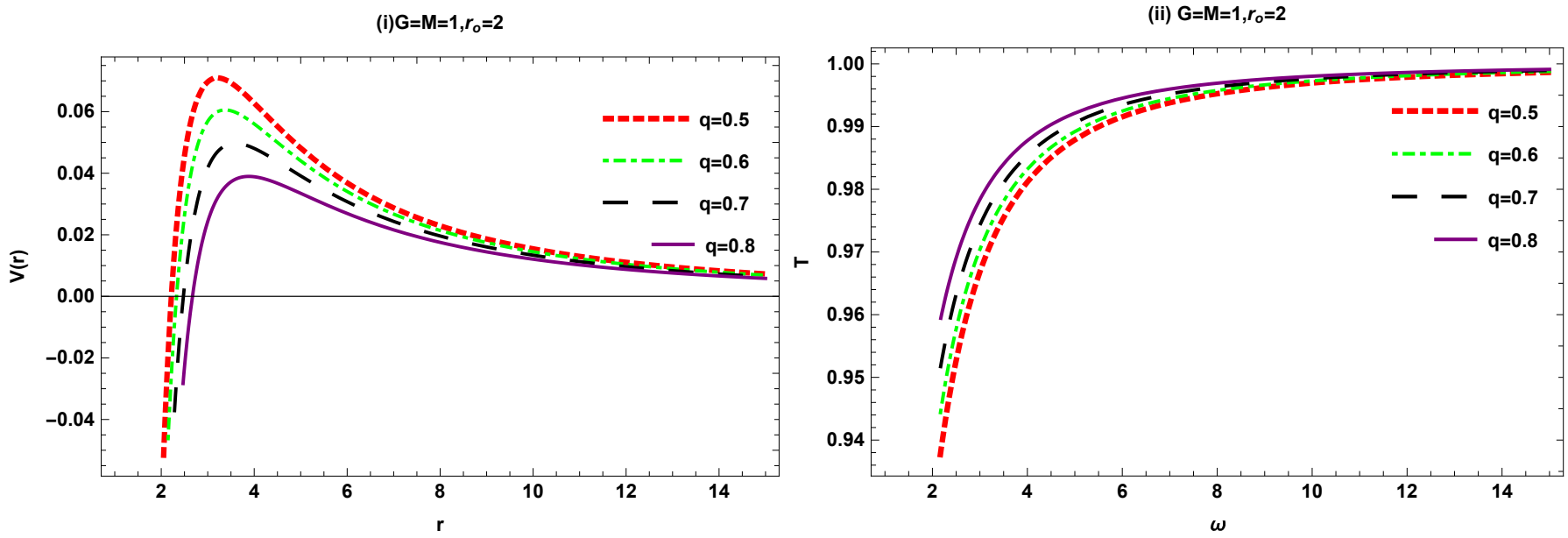

Figure 15: (i) indicates the potential behaviour with $l=1$ and (ii) indicates the corresponding greybody factor bound of electric $\mathrm{BH}$.

- We analyze that the value of the potential with $l=1$ starts increasing when the value of the electric charge $q$ decreases and the corresponding bound of the greybody factor becomes lower and it becomes difficult for the wave to transmit through higher potential. 
(i) $\mathrm{G}=\mathrm{M}=1, \beta=5$

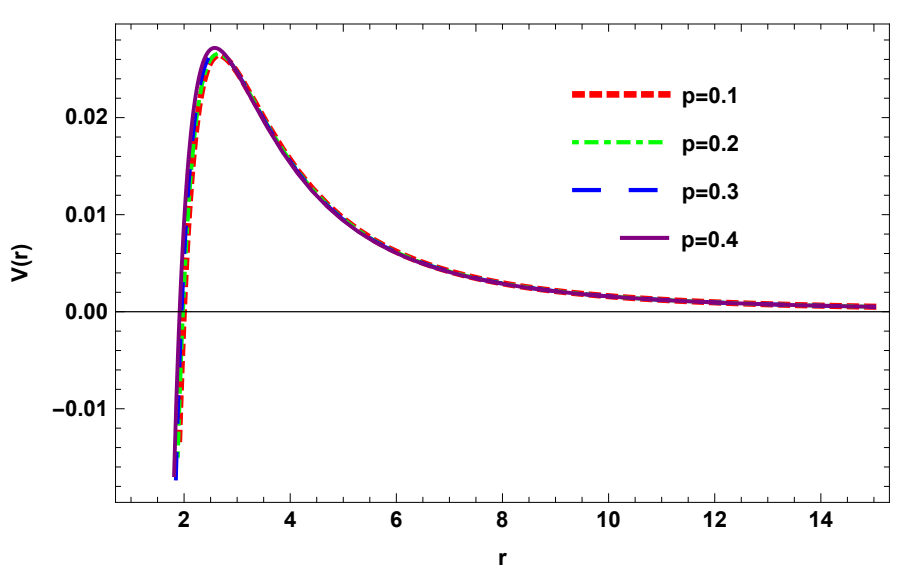

(ii) $\mathrm{G}=\mathrm{M}=1, \beta=5$

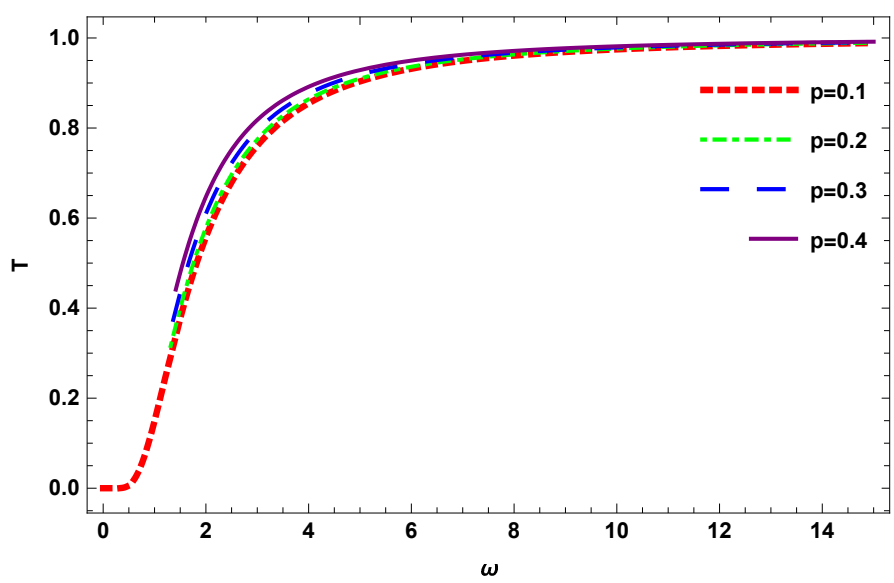

Figure 16: (i) indicates the potential behaviour with $l=0$ and (ii) indicates the regarding greybody factor bound of magnetic $\mathrm{BH}$.

- We analyze that the value of the potential with $l=0$ starts increasing when the value of the electric charge $q$ increases and bound of the greybody factor becomes lower and it becomes difficult for the wave to transmit through the high value of the potential.
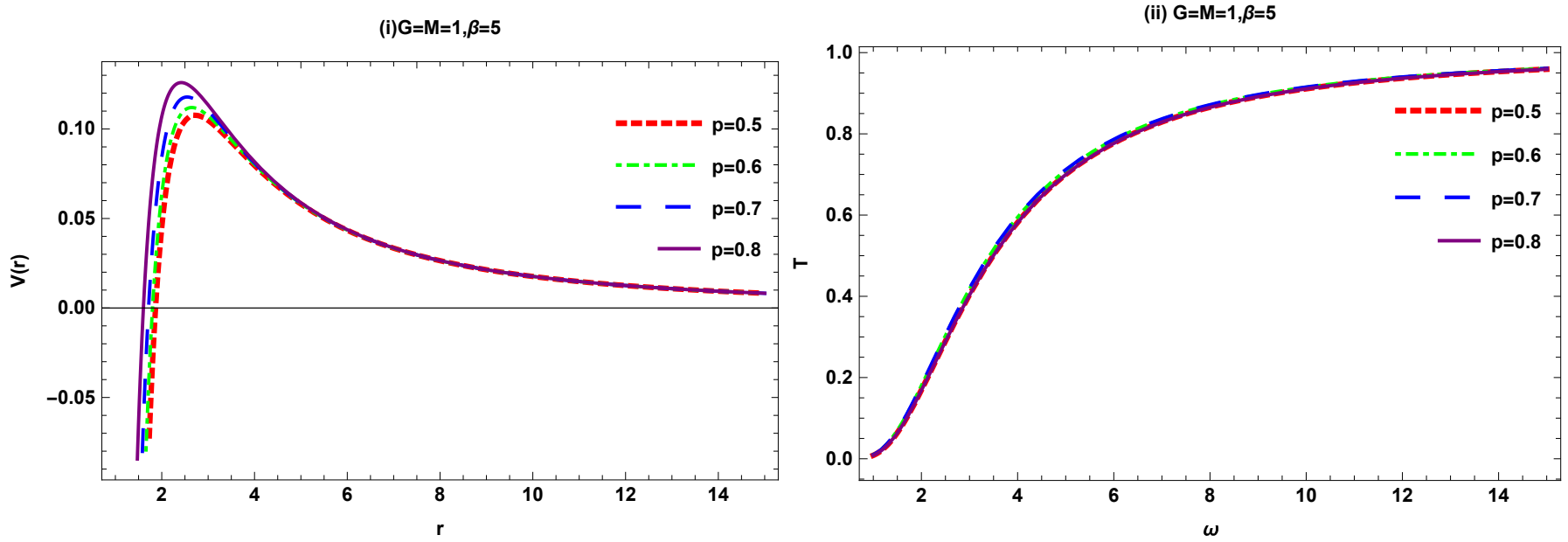

Figure 17: (i) indicates the potential behaviour with $l=1$ and (ii) indicates the regarding greybody factor bound of magnetic $\mathrm{BH}$.

- We investigate that the potential with $l=1$ increases as the value of the magnetic charge $p$ increases and the corresponding bound becomes lower and is difficult for the wave to pass through the high value of the potential.

\section{CONCLUSION}

In our analysis, we have analyzed the NLED model to calculate the bending angles in non-plasma and plasma mediums and greybody factor bounds for electric and magnetic BHs. The results are obtained as follows.

\section{Bending Angle $\alpha$}

\section{(i) Non-plasma Medium}

The bending angles for electric and magnetic BHs are obtained by using the value of $\mathcal{K}$ as follows

$$
\begin{aligned}
\alpha & \simeq \frac{4 G M}{b}+\frac{G^{2} M \pi q^{2}}{r_{o}^{2} b}+\mathcal{O}\left(M^{2}, q^{4}\right), \\
\alpha & \simeq \frac{4 G M}{b}-\frac{3 G p^{2} \pi}{4 b^{2}}+\beta \frac{7 G p^{4} \pi}{384 b^{6}}+\mathcal{O}\left(G^{2}, M^{2}\right) .
\end{aligned}
$$


We observed that the obtained bending angle for electric $\mathrm{BH}$ depends on the impact parameter $b$, mass of the $\mathrm{BH}$, parameter $r_{o}$ and the electric charge $q$ and for the magnetic $\mathrm{BH}$ bending angle depends on the impact parameter $b$, mass of the $\mathrm{BH}, \beta$ and magnetic charge $p$. We proved that the bending angles for electric and magnetic BHs reduces to Schwarzschild $\mathrm{BH}$ bending angle in non-plasma medium upto the order one of $M$ if we neglect the electric and magnetic charges.

\section{(ii) Plasma Medium}

We also discussed the bending angles of these BHs in plasma medium by using the same technique as in non-plasma medium. The Bending angles we have obtained for electric and magnetic BHs in plasma medium respectively as follows

$$
\begin{aligned}
\alpha & \simeq \frac{4 G M}{b}+\frac{G^{2} M \pi q^{2}}{r_{o}^{2} b}+\frac{2 G M \omega_{e}^{2}}{b \omega_{\infty}^{2}}-\frac{G^{2} M \pi q^{2} \omega_{e}^{2}}{2 r_{o}^{2} b \omega_{\infty}^{2}}+\mathcal{O}\left(M^{2}, q^{4}\right) . \\
\alpha & \simeq \frac{4 G M}{b}-\frac{3 G p^{2} \pi}{4 b^{2}}+\beta \frac{7 G p^{4} \pi}{384 b^{6}}+\frac{2 G M \omega_{e}^{2}}{b \omega_{\infty}^{2}}-\frac{G p^{2} \pi \omega_{e}^{2}}{2 b^{2} \omega_{\infty}^{2}} \\
& +\beta \frac{G p^{4} \pi \omega_{e}^{2}}{64 b^{6} \omega_{\infty}^{2}}+\mathcal{O}\left(G^{2}, M^{2}\right) .
\end{aligned}
$$

We have investigated that the obtained bending angle $\alpha$ for electric $\mathrm{BH}$ depends on the impact parameter $b$, mass of the $\mathrm{BH}$, parameter $r_{o}$ and the electric charge $q$ of the $\mathrm{BH}$ and for magnetic $\mathrm{BH}$ depends on the impact parameter $b$, mass of the $\mathrm{BH}$, parameter $\beta$ and magnetic charge $p$. We proved that the bending angles for electric and magnetic BHs reduces to Schwarzschild $\mathrm{BH}$ bending angle in non-plasma medium upto the order one of $M$ if we neglect the electric and magnetic BHs charges. If we remove the plasma effect then the bending angles in plasma medium reduces to the bending angles for both electric and magnetic BHs in non-plasma medium.

\section{Graphically}

After computing the bending angles, we also analyzed the graphical behaviour of the bending angles for both electric and magnetic charged BHs in both non-plasma and plasma mediums. We also observed that the graphical behaviour exhibits similar results in both non-plasma and plasma mediums. These results are described as follows

\section{(i) For Electric Black Hole}

\section{- Bending angle $\alpha$ versus impact parameter $b$ :}

For the fixed values of $G=M=1$ and $r_{o}=2$, for the small values of $q$ bending angle $\alpha$ is uniformly constant and for the large values of $q$ bending angle $\alpha$ is increasing gradually.

\section{- Bending angle $\alpha$ versus electric charge $q$ :}

For the fixed values of $G=M=1$ and $r_{o}=2$, for the small values of $b$ bending angle $\alpha$ is decreasing and for the large values of $b$ bending angle $\alpha$ firslty increasing and then start decreasing.

\section{(ii) For Magnetic Black Hole}

\section{- Bending angle $\alpha$ versus impact parameter $b$ :}

For the small values of $p(G=M=1$ and $\beta=5)$ bending angle $\alpha$ is rapidly decreasing and for the large values of $p$ ( $G=M=1$ and $\beta=10$ ) bending angle $\alpha$ is decreasing and then goes to infinity. For the small values of $\beta(G=M=1$ and $p=0.45)$ bending angle $\alpha$ is constantly decreasing and for the large values of $\beta(G=M=1$ and $p=10)$ bending angle $\alpha$ is decreasing negatively and then increasing goes to infinity.

\section{- Bending angle $\alpha$ versus magnetic charge $p$ :}

For the small values of $b(G=M=1$ and $\beta=5)$ firstly the bending angle $\alpha$ is increasing and the start decreasing and for the large values of $\beta(G=M=1$ and $b=5)$ bending angle $\alpha$ is negatively increasing.

\section{- Bending angle $\alpha$ versus parameter $\beta$ :}

For the fixed values of $G=M=1$ and $p=5$, for the small values of $b$ bending angle $\alpha$ is decreasing and for the large values of $b$, bending angle $\alpha$ also shows the decreasing behaviour. 


\section{Greybody Factor Bounds $T$}

We calculated the greybody factor bounds for both electric and magnetic BHs. In order to obtain the greybody bounds, we graphically determined the horizon for magnetic $\mathrm{BH}$ and for electric $\mathrm{BH}$ we calculate the horizon numerically.

\section{(i) For Electric Black Hole}

The bound on the greybody factor in the case of electric BH obtained as

$$
T \geq \frac{1}{\cosh ^{2}}\left[\frac{1}{2 \omega}\left(\frac{\left(G \pi q^{2}-2 r_{o}^{2}\right)^{2}}{16 r_{o}^{4} G M}-\frac{l(l+1)\left(G \pi q^{2}-2 r_{o}^{2}\right)}{4 r_{o}^{2} G M}\right)\right] .
$$

The obtained bound for the greybody factor of the electric BH depends on the mass of the $\mathrm{BH}, r_{o}$ and the electric charge $q$ of the $\mathrm{BH}$. While in the absence of the electric charge $q$, the above bound for the electric $\mathrm{BH}$ reduces into the form

$$
T \geq \frac{1}{\cosh ^{2}}\left[\frac{2 l(l+1)+1}{8 \omega G M}\right]
$$

which is the lower bound on the greybody factor of the Schwarzschild BH.

(ii) For Magnetic Black Hole

In the case of magnetic $\mathrm{BH}$, the derived bound on the greybody factor is defined as follows

$$
T \geq \frac{1}{\cosh ^{2}}\left[\frac{3.24649 G M-3.89969 G p^{2}+1.8018 l(l+1)+0.880747 G p^{4} \beta}{2 \omega}\right] .
$$

The bound for the greybody factor of magnetic $\mathrm{BH}$ depend on the mass of the $\mathrm{BH}$, magnetic charge $p$ and parameter $\beta$.

\section{Graphical Analysis of Greybody Bound}

At the end, we investigated the graphical results of the greybody bounds for different values of electric and magnetic charges. We noticed that the greybody bound relies on the greatest worth of the potential. We have analyzed that for the small value of electric charge $q$ of electric $\mathrm{BH}$, the value of the potential increases and the bound decreases. In case of magnetic $\mathrm{BH}$, the potential increases as the value of the magnetic charge $p$ increases and the value of the bound decreases and it becomes difficult for the waves to passed through the highest potential.

[1] A. Einstein, Science 84, 506-507 (1936).

[2] C. A. R. Herdeiro and J. P. S. Lemos, [arXiv:1811.06587 [physics.hist-ph]].

[3] B. P. Abbott et al. [LIGO Scientific and Virgo], Phys. Rev. Lett. 116, no.13, 131103 (2016).

[4] K. Akiyama et al. [Event Horizon Telescope], Astrophys. J. Lett. 875, L1 (2019).

[5] P. V. P. Cunha and C. A. R. Herdeiro, Gen. Rel. Grav. 50, no.4, 42 (2018).

[6] M. Bartelmann and P. Schneider, Phys. Rept. 340, 291-472 (2001).

[7] J. Soldner, Ueber die Ablenkung eines Lichtstrals von seiner geradlinigen Bewegung, durch die Attraktion eines Weltkörpers, an welchem er nahe vorbei geht. Berliner Astronomisches Jahrbuch,161-172.cc (1804).

[8] F. W. Dyson, A. S. Eddington and C. Davidson, Phil. Trans. Roy. Soc. Lond. A 220, 291-333 (1920).

[9] E. F. Eiroa, G. E. Romero and D. F. Torres, Phys. Rev. D 66, 024010 (2002).

[10] C. R. Keeton, C. S. Kochanek and E. E. Falco, Astrophys. J. 509, 561-578 (1998).

[11] M. Sharif and S. Iftikhar, Astrophys. Space Sci. 361, no.1, 36 (2016).

[12] K. S. Virbhadra, D. Narasimha and S. M. Chitre, Astron. Astrophys. 337, 1-8 (1998).

[13] A. F. Zakharov, Int. J. Mod. Phys. D 27, no.06, 1841009 (2018).

[14] K. S. Virbhadra and G. F. R. Ellis, Phys. Rev. D 65, 103004 (2002)

[15] R. Shaikh, P. Kocherlakota, R. Narayan and P. S. Joshi, Mon. Not. Roy. Astron. Soc. 482, no.1, 52-64 (2019).

[16] S. U. Islam, R. Kumar and S. G. Ghosh, JCAP 09, 030 (2020)

[17] R. Kumar, S. G. Ghosh and A. Wang, Phys. Rev. D 101, no.10, 104001 (2020)

[18] T. Manna, F. Rahaman, S. Molla, J. Bhadra and H. H. Shah, Gen. Rel. Grav. 50, no.5, 54 (2018).

[19] P. K. F. Kuhfittig, Eur. Phys. J. C 74, no.99, 2818 (2014).

[20] F. Rahaman, M. Kalam and S. Chakraborty, Chin. J. Phys. 45, 518 (2007).

[21] G. W. Gibbons and M. C. Werner, Class. Quant. Grav. 25, 235009 (2008).

[22] M. C. Werner, Gen. Rel. Grav. 44, 3047-3057 (2012).

[23] G. Crisnejo and E. Gallo, Phys. Rev. D 97, no.12, 124016 (2018).

[24] A. Ishihara, Y. Suzuki, T. Ono, T. Kitamura and H. Asada, Phys. Rev. D 94, no.8, 084015 (2016)

[25] A. Ishihara, Y. Suzuki, T. Ono and H. Asada, Phys. Rev. D 95, no.4, 044017 (2017).

[26] A. Övgün, Phys. Rev. D 98, no.4, 044033 (2018).

[27] A. Övgün, Phys. Rev. D 99, no.10, 104075 (2019).

[28] A. Övgün, Universe 5, no.5, 115 (2019). 
[29] K. Jusufi, M. C. Werner, A. Banerjee and A. Övgün, Phys. Rev. D 95, no.10, 104012 (2017).

[30] K. de Leon and I. Vega, Phys. Rev. D 99, no.12, 124007 (2019).

[31] K. Jusufi and A. Övgün, Phys. Rev. D 97, no.2, 024042 (2018).

[32] T. Ono, A. Ishihara and H. Asada, Phys. Rev. D 96, no.10, 104037 (2017)

[33] K. Jusufi, A. Ovgün and A. Banerjee, Phys. Rev. D 96, no.8, 084036 (2017).

[34] A. Övgün, G. Gyulchev and K. Jusufi, Annals Phys. 406, 152-172 (2019).

[35] H. Arakida, Gen. Rel. Grav. 50, no.5, 48 (2018).

[36] K. Jusufi and A. Ovgün, Phys. Rev. D 97, no.6, 064030 (2018).

[37] T. Ono, A. Ishihara and H. Asada, Phys. Rev. D 98, no.4, 044047 (2018).

[38] K. Jusufi, A. Övgün, J. Saavedra, Y. Vásquez and P. A. González, Phys. Rev. D 97, no.12, 124024 (2018).

[39] T. Ono, A. Ishihara and H. Asada, Phys. Rev. D 99, no.12, 124030 (2019).

[40] W. Javed, R. Babar and A. Övgün, Phys. Rev. D 99, no.8, 084012 (2019).

[41] K. Jafarzade, M. Kord Zangeneh and F. S. N. Lobo, JCAP 04, 008 (2021).

[42] K. Takizawa, T. Ono and H. Asada, Phys. Rev. D 101, no.10, 104032 (2020).

[43] Y. Kumaran and A. Övgün, Chin. Phys. C 44, no.2, 025101 (2020).

[44] Z. Li and A. Övgün, Phys. Rev. D 101, no.2, 024040 (2020).

[45] Z. Li, G. Zhang and A. Övgün, Phys. Rev. D 101, no.12, 124058 (2020).

[46] A. Övgün, Turk. J. Phys. 44, no.5, 465-471 (2020).

[47] Y. Kumaran and A. Övgün, Turk. J. Phys. 45, 247-267 (2021).

[48] M. Okyay and A. Övgün, JCAP 01, no.01, 009 (2022).

[49] R. C. Pantig, P. K. Yu, E. T. Rodulfo and A. Övgün, Annals of Physics 436, 168722 (2022).

[50] K. Takizawa, T. Ono and H. Asada, Phys. Rev. D 102, no.6, 064060 (2020).

[51] T. Ono and H. Asada, Universe 5, no.11, 218 (2019).

[52] Q. M. Fu, L. Zhao and Y. X. Liu, Phys. Rev. D 104, no.2, 024033 (2021).

[53] H. El Moumni, K. Masmar and A. Övgün, [arXiv:2008.06711 [gr-qc]].

[54] W. Javed, R. Babar and A. Övgün, Phys. Rev. D 100, no.10, 104032 (2019).

[55] W. Javed, J. Abbas and A. Övgün, Eur. Phys. J. C 79, no.8, 694 (2019).

[56] W. Javed, j. Abbas and A. Övgün, Phys. Rev. D 100, no.4, 044052 (2019).

[57] W. Javed, A. Hamza and A. Övgün, Phys. Rev. D 101, no.10, 103521 (2020).

[58] W. Javed, M. B. Khadim, A. Övgün and J. Abbas, Eur. Phys. J. Plus 135, no.3, 314 (2020).

[59] W. Javed, M. B. Khadim and A. Övgün, Eur. Phys. J. Plus 135, no.7, 595 (2020).

[60] W. Javed, A. Hamza and A. Övgün, Mod. Phys. Lett. A 35, no.39, 2050322 (2020).

[61] W. Javed, A. Hamza and A. Övgün, Universe 7, no.10, 385 (2021).

[62] W. Javed, J. Abbas, Y. Kumaran and A. Övgün, Int. J. Geom. Meth. Mod. Phys. 18, no.01, 2150003 (2021).

[63] W. Javed, J. Abbas and A. Övgün, Annals Phys. 418, 168183 (2020).

[64] S. Hensh, A. Abdujabbarov, J. Schee and Z. Stuchlík, Eur. Phys. J. C 79, no.6, 533 (2019).

[65] S. W. Hawking, Commun. Math. Phys. 43, 199-220 (1975) [erratum: Commun. Math. Phys. 46, 206 (1976)].

[66] S. Fernando, Gen. Rel. Grav. 37, 461-481 (2005).

[67] G. Panotopoulos and A. Rincón, Phys. Rev. D 97, no.8, 085014 (2018).

[68] J. M. Maldacena and A. Strominger, Phys. Rev. D 55, 861-870 (1997).

[69] D. Ida, K. y. Oda and S. C. Park, Phys. Rev. D 67, 064025 (2003) [erratum: Phys. Rev. D 69, 049901 (2004)].

[70] M. Cvetic and F. Larsen, Phys. Rev. D 56, 4994-5007 (1997).

[71] T. Harmark, J. Natario and R. Schiappa, Adv. Theor. Math. Phys. 14, no.3, 727-794 (2010).

[72] S. S. Gubser and I. R. Klebanov, Phys. Rev. Lett. 77, 4491-4494 (1996).

[73] I. R. Klebanov and S. D. Mathur, Nucl. Phys. B 500, 115-132 (1997).

[74] H. W. Lee and Y. S. Myung, Phys. Rev. D 58, 104013 (1998).

[75] S. Chen and J. Jing, Phys. Lett. B 691, 254-260 (2010).

[76] R. A. Konoplya and A. F. Zinhailo, Phys. Lett. B 810, 135793 (2020).

[77] P. Boonserm and M. Visser, Annals Phys. 323, 2779-2798 (2008).

[78] P. Boonserm, [arXiv:0907.0045 [math-ph]].

[79] P. Boonserm and M. Visser, Phys. Rev. D 78, 101502 (2008).

[80] T. Ngampitipan and P. Boonserm, Int. J. Mod. Phys. D 22, 1350058 (2013).

[81] P. Boonserm, T. Ngampitipan and P. Wongjun, Eur. Phys. J. C 78, no.6, 492 (2018).

[82] P. Boonserm, T. Ngampitipan and M. Visser, JHEP 03, 113 (2014).

[83] P. Boonserm, A. Chatrabhuti, T. Ngampitipan and M. Visser, J. Math. Phys. 55, 112502 (2014).

[84] T. Ngampitipan and P. Boonserm, J. Phys. Conf. Ser. 435, 012027 (2013).

[85] P. Boonserm, T. Ngampitipan and P. Wongjun, Eur. Phys. J. C 79, no.4, 330 (2019).

[86] S. H. Mazharimousavi and M. Halilsoy, Annals Phys. 433, 168579 (2021).

[87] A. Övgün, Eur. Phys. J. C 77, no.2, 105 (2017).

[88] A. Övgün, G. Leon, J. Magaña and K. Jusufi, Eur. Phys. J. C 78, no.6, 462 (2018).

[89] D. P. Sorokin, [arXiv:2112.12118 [hep-th]].

[90] H. B. Benaoum and A. Ovgun, Class. Quant. Grav. 38, no.13, 135019 (2021).

[91] P. Sarkar, P. Kumar Das and G. C. Samanta, Phys. Scripta 96, no.6, 065305 (2021).

[92] G. Otalora, A. Övgün, J. Saavedra and N. Videla, JCAP 06, 003 (2018). 
[93] S. H. Mazharimousavi and M. Halilsoy, Annalen Phys. 531, no.12, 1900236 (2019).

[94] S. I. Kruglov, Int. J. Mod. Phys. A 32, no.23n24, 1750147 (2017)

[95] S. I. Kruglov, Universe 4, no.5, 66 (2018)

[96] S. I. Kruglov, Annals Phys. 378, 59-70 (2017).

[97] M. S. Rad, S. H. Hendi, K. Matsuno and A. Sheykhi, Annals Phys. 363, 485-495 (2015)

[98] E. Ayon-Beato and A. Garcia, Phys. Rev. Lett. 80, 5056-5059 (1998).

[99] K. A. Bronnikov, Phys. Rev. D 63, 044005 (2001).

[100] E. Ayon-Beato and A. Garcia, Phys. Lett. B 493, 149-152 (2000).

[101] E. Ayon-Beato and A. Garcia, Phys. Lett. B 464, 25 (1999).

[102] S. A. Hayward, Phys. Rev. Lett. 96, 031103 (2006).

[103] S. Ansoldi, P. Nicolini, A. Smailagic and E. Spallucci, Phys. Lett. B 645, 261-266 (2007).

[104] C. Bambi and L. Modesto, Phys. Lett. B 721, 329-334 (2013).

[105] B. Toshmatov, B. Ahmedov, A. Abdujabbarov and Z. Stuchlik, Phys. Rev. D 89, no.10, 104017 (2014).

[106] J. P. S. Lemos and V. T. Zanchin, Phys. Rev. D 83, 124005 (2011).

[107] G. J. Olmo and D. Rubiera-Garcia, Phys. Rev. D 84, 124059 (2011).

[108] V. P. Frolov, Phys. Rev. D 94, no.10, 104056 (2016)

[109] M. Born and L. Infeld, Proc. Roy. Soc. Lond. A 144, no.852, 425-451 (1934). 